\title{
Remembering specific features of emotional events across time: The role of REM sleep and prefrontal theta oscillations
}

\author{
Marie Roxanne Sopp $^{1} \cdot$ Tanja Michael $^{1} \cdot$ Hans-Günter Weeß ${ }^{2} \cdot$ Axel Mecklinger $^{3}$
}

Published online: 23 October 2017

(C) Psychonomic Society, Inc. 2017

\begin{abstract}
When an episode of emotional significance is encountered, it often results in the formation of a highly resistant memory representation that is easily retrieved for many succeeding years. Recent research shows that beyond generic consolidation processes, rapid eye movement (REM) sleep importantly contributes to this effect. However, the boundary conditions of consolidation processes during REM sleep, specifically whether these extend to source memory, have not been examined extensively. The current study tested the effects of putative consolidation processes emerging during REM sleep and slow wave sleep (SWS) on item and source memory of negative and neutral images, respectively. Results demonstrate superior emotional relative to neutral item memory retention after both late night REM sleep and early night SWS. Emotional source memory, on the other hand, exhibited an attenuated decline following late night REM sleep, whereas neutral source memory was selectively preserved across early night SWS. This pattern of results suggests a selective preservation of emotional source memory during REM sleep that is functionally dissociable from SWS-dependent reprocessing of
\end{abstract}

Electronic supplementary material The online version of this article (https://doi.org/10.3758/s13415-017-0542-8) contains supplementary material, which is available to authorized users.

Marie Roxanne Sopp

roxanne.sopp@uni-saarland.de

1 Division of Clinical Psychology and Psychotherapy, Department of Psychology, Saarland University, Campus A1.3, 66123 Saarbruecken, Germany

2 Interdisciplinary Center of Sleep, Pfalzklinikum, Klinikum für Psychiatrie und Neurologie AdöR, Klingenmünster, Germany

3 Experimental Neuropsychology Unit, Department of Psychology, Saarland University, Saarbruecken, Germany neutral source memory. This was further substantiated by a neurophysiological dissociation: Postsleep emotional source memory was selectively correlated with frontal theta lateralization (REM sleep), whereas postsleep neutral item memory was correlated with SWS spindle power. As such, the present results contribute to a more comprehensive characterization of sleep-related consolidation mechanisms underlying emotional and neutral memory retention. Subsidiary analysis of emotional reactivity to previously encoded material revealed an enhancing rather than attenuating effect of late night REM sleep on emotional responses.

Keywords Emotion · Item memory $\cdot$ Source memory $\cdot$ SWS Sleep spindles $\cdot$ Right-frontal theta lateralization

Sleep has been shown to have a profound effect on episodic memory consolidation that goes beyond mere protection from retroactive interference (Diekelmann \& Born, 2010). Recent research affirms this notion by demonstrating the remarkable selectivity with which sleep-inherent processes operate to yield an enhanced retention of memory representations that are of specific future relevance (Barner, Seibold, Born, \& Diekelmann, 2017; Bennion, Payne, \& Kensinger, 2016; Diekelmann, Wilhelm, Wagner, \& Born, 2013; Fischer \& Born, 2009; Iglói, Gaggioni, Sterpenich, \& Schwartz, 2015; Lo, Bennion, \& Chee, 2016; Rauchs et al., 2011; Saletin, Goldstein, \& Walker, 2011; Stickgold \& Walker, 2013; Studte, Bridger, \& Mecklinger, 2017; van Dongen, Thielen, Takashima, Barth, \& Fernández, 2012; Wilhelm et al., 2011). These differential retention benefits are assumed to rely on selection processes during instantaneous encoding of highly relevant information (Heib et al., 2015; see Baran, Daniels, \& Spencer, 2013; Tucker, Tang, Uzoh, Morgan, \& Stickgold, 2011, for accounts suggesting that these effects may be less 
evident when learning is enforced by feedback or multiple encoding cycles). Contemporary models (Diekelmann \& Born, 2010) assume that selective sleep-related consolidation effects are further established during slow wave sleep (SWS), which is believed to offer a window of opportunity for reactivations of newly acquired memory representations. These reactivations are presumed to originate from the hippocampus, which is reflected on an oscillatory level by the propagation of sharp-wave/ripple (SPW-R) complexes. These SPW-Rs are believed to enclose compressed hippocampal reactivations and may thus enable a gradual redistribution toward long-term storage in the neocortex (Buzsáki, 2015). This process is mediated by thalamocortical sleep spindles (11-15 $\mathrm{Hz})$, whereas neocortical slow oscillations $(<1 \mathrm{~Hz})$ are believed to drive the initial reactivation of memory representations in the hippocampus (Diekelmann \& Born, 2010).

Interestingly, sleep's selectivity in memory consolidation also pertains to different aspects of episodic memory, specifically, to item and associative memory processes (van der Helm, Gujar, Nishida, \& Walker, 2011). Although item memory, which refers to the recognition of a stimulus from a prior study episode, seems to benefit only mildly from SWS, associative memory appears to benefit profoundly from SWS in the consolidation phase (Mednick et al., 2013; Studte, Bridger, \& Mecklinger, 2015). Associative memory refers to the retrieval of contextual or source features of a study episode and thus entails the binding of multiple features to represent an event (Yonelinas, 2002). These binding processes at the encoding and retrieval stage have been found to critically rely on the hippocampus (Staresina \& Davachi, 2009; Yonelinas, 2013). Consequently, the observation that SWS selectively enhances associative memory aligns well with the active system consolidation account (Diekelmann \& Born, 2010), which assumes that reactivations during SWS emerge specifically from hippocampal processes. Item memory, on the other hand, is supported by two distinct memory processes according to dual-process models of recognition memory (Yonelinas, 2002). These retrieval processes are referred to as recollection and familiarity and are assumed to engage distinct neurophysiological circuits, with only the former relying on the retrieval of hippocampal-bound associative information. In accordance with the evidence of a selective involvement in associative memory, sleep has thus been found to enhance recollection ("remembering") rather than familiarity ("knowing") in item memory tests (Daurat, Terrier, Foret, \& Tiberge, 2007; Drosopoulos, Wagner, \& Born, 2005). Critically, the selective involvement in associative memory is also evident on a neurophysiological level: As previously mentioned, the process of redistribution in system consolidation is assumed to be reflected on an oscillatory level by the temporal coupling of hippocampal SWRs and thalamocortical sleep spindles (Staresina et al., 2015). This process can be partially ascertained by examining EEG activity in the spindle frequency range $(11-15 \mathrm{~Hz})$ during SWS (Mednick et al., 2013; Studte et al., 2015), which has yielded complemental evidence of highly specific correlations between postsleep associative memory performance and spindle density during SWS (Studte et al., 2015). Despite these converging findings across different approaches, evidence is not entirely unequivocal as to the selective redistribution of hippocampusdependent memories during sleep-related system consolidation. As such, item memory performance has also been shown to benefit from SWS (see, e.g., Schönauer, Pawlizki, Köck, \& Gais, 2014). However, as item and associative memory tests may not yield process-pure measures of underlying retrieval processes (see above), this could partially account for inconsistent findings between studies employing different task procedures (Yonelinas, 2002).

Sleep-related effects on episodic memory formation have been found to exhibit another highly distinctive feature, which pertains to the selective consolidation of emotionally significant episodes. Emotional — as compared to neutral—stimuli generally exhibit preferential consolidation over time (Yonelinas \& Ritchey, 2015), but this effect is markedly enhanced by sleep (Stickgold \& Walker, 2013). Studies investigating these effects employ a variety of different designs (e.g., sleep deprivation, nap sleep, nocturnal sleep) with varying degrees of experimental control (e.g., lab-monitored vs. home-based sleep), yielding partially inconsistent results (see Atienza \& Cantero, 2008; Baran, PaceSchott, Ericson, \& Spencer, 2012; Cellini, Torre, Stegagno, \& Sarlo, 2016; Sterpenich et al., 2007). Nevertheless, a majority of findings demonstrate enhanced retention of emotional stimuli across sleep (Cunningham et al., 2014b; Groch, Wilhelm, Diekelmann, \& Born, 2013; Groch, Zinke, Wilhelm, \& Born, 2015; Hu, Stylos-Allan, \& Walker, 2006; Nishida, Pearsall, Buckner, \& Walker, 2009; Payne, Chambers, \& Kensinger, 2012; Payne \& Kensinger, 2011; Payne, Stickgold, Swanberg, \& Kensinger, 2008; Prehn-Kristensen et al., 2009; PrehnKristensen et al., 2013; Wagner, Gais, \& Born, 2001).This enhancement has been found to co-occur with profound changes in the neural circuits underlying emotional memory. After sleep, recollection of emotional material is associated with enhanced functional connectivity between the hippocampus and medial prefrontal cortex (Cairney, Durrant, Power, \& Lewis, 2015; Sterpenich et al., 2007) as well as attenuated responding of the amygdala (Rosales-Lagarde et al., 2012; Sterpenich et al., 2007). These findings have been interpreted to reflect a decline of emotional reactivity that co-occurs with enhanced recollection of emotional stimuli across sleep (Walker \& van der Helm, 2009; but see Baran et al., 2012; Groch et al., 2013; Payne \& Kensinger, 2011; Sterpenich et al., 2009; Werner, Schabus, Blechert, Kolodyazhniy, \& Wilhelm, 2015).

Despite substantial evidence of a selective consolidation of emotional memories during sleep, remarkably little is known about the exact mechanism by which sleep modulates the consolidation of emotional memories. In contrast to the aforementioned effects of SWS on associative memory, emotional memory enhancements have been brought into association 
with REM sleep physiology (Groch et al., 2013; Groch, et al., 2015; Nishida et al., 2009; Payne et al., 2012; Wagner et al., 2001; Wiesner et al., 2015). Certain inconsistencies in the current state of research (see, e.g., Baran et al., 2012; Cairney, Durrant, Jackson, \& Lewis, 2014; Cairney et al., 2015) may be explained by prevailing efforts to link emotional memory retention with macrostructural features of REM sleep architecture (e.g., duration in minutes or $\%$ total sleep time [TST]). As the length of time spent in a particular sleep stage may not accurately reflect on the processes which occur in this time frame (e.g., Datta \& O'Malley, 2013), it is important to examine microstructural REM sleep features as potential markers of sleep-related consolidation. In line with this conception, preliminary findings demonstrate that emotional memory reprocessing during REM sleep is associated with EEG theta activity $(4-7 \mathrm{~Hz})$ and specifically with the extent of right-frontal theta lateralization (Nishida et al., 2009; Prehn-Kristensen et al., 2013; but see Ackermann, Hartmann, Papassotiropoulos, de Quervain, \& Rasch, 2015, for a large-scale field study failing to replicate this association). The notion of a pivotal role of theta oscillations has been additionally substantiated by experimental work in animal models. Rodent studies demonstrate a significant impact of theta coherence between the amygdala and hippocampus on the retention of conditioned fear responses as well as an impairment in contextual memory consolidation when theta activity is selectively attenuated (Boyce, Glasgow, Williams, \& Adamantidis, 2016; Hutchison \& Rathore, 2015; Popa, Duvarci, Popescu, Léna, \& Paré, 2010).

Beyond the neurophysiological processes underlying emotional memory enhancements across periods of sleep, only a few studies have explored whether the beneficial effects of REM sleep are restricted to emotional item memory or if they extend to emotional associative memory. Initial studies did not find selective facilitating effects of sleep on associative memory for emotional items (Cairney, Durrant, Hulleman, \& Lewis, 2013; Lewis, Cairney, Manning, \& Critchley, 2011) but demonstrated a uniform enhancement of associative memory after sleep irrespective of stimulus valence (Lewis et al., 2011). This is in line with the predictions of the active system consolidation hypothesis and suggests that SWS-inherent processing mechanisms are equally instated for emotional and neutral associations. Interestingly, two recent studies demonstrate that sleepdependent processing is only associated with enhanced associative recognition of neutral but not emotional images (Alger \& Payne, 2016; Groch et al., 2015), thereby indicating that SWSdependent consolidation of associative memory may only emerge for neutral stimuli. Due to the inconclusiveness of empirical findings, it is therefore an open issue whether emotional associative memory, similar to neutral associative memory, is supported by SWS or whether it benefits from REM sleepdependent processing, similar to emotional item memory. A potentially enhancing influence of REM sleep-related consolidation on emotional associative memory may be restricted to specific circumstances (Chiu, Dolcos, Gonsalves, \& Cohen, 2013; Kensinger, 2009; Mather, 2007). It is thus currently assumed that arousal has an enhancing effect on succeeding stages of associative memory formation only when the to-be-retained feature is of functional significance during item processing, or presented in close spatiotemporal proximity to the arousing item (hence referred to as "intrinsic" features; Kensinger, 2009; Mather, 2007). "Extrinsic" features, which are conceptually or perceptually delimitable from the arousing item during encoding, may conversely be susceptible to effects of attentional narrowing (Easterbrook, 1959), and thus may not benefit from preferential processing of emotional material during subsequent stages of memory formation. Consequently, REM sleep may promote the consolidation of emotional associative memory only when associative features are perceived as being "intrinsic" to the stimulus. Moreover, memory processes involving emotional material have been frequently found to implicate the recruitment of a different network of neural structures, including the amygdala and the parahippocampal area (Kensinger \& Corkin, 2004; Murray \& Kensinger, 2013). This neural network, in turn, exhibits markedly enhanced activation during REM sleep (Walker \& van der Helm, 2009). Thus, REM sleep-dependent consolidation of emotional associative memory may act independently of SWS-dependent system-consolidation processes. Indeed, initial evidence suggests that retrieval of emotional associations following sleep is related to an enhanced response of the emotional memory network as compared to retrieval after sustained wakefulness (Lewis et al., 2011).

The current study aimed to explore the role of REM sleep and SWS in the consolidation of emotional item and associative memory. In order to contrast the effects of SWS and REM sleep, participants were subjected to a between-subjects split-night manipulation, which entailed a consolidation interval of either early night SWS-rich or late night REM sleep-rich sleep. Memory performance was ascertained in a combined procedure probing item memory and associative memory by means of a source memory paradigm. Participants encoded emotional (as defined by high arousal and low valence) and neutral images from various semantic categories at different screen locations. Thus, accurately recognizing images served as our measure of item memory, whereas the successful retrieval of the source feature (i.e., the images' screen location) was employed as a measure of associative memory and hence will be referred to as source memory. In addition, we chose to investigate item memory with a remember/ know procedure (Rajaram, 1993), which allows differentiating between familiarity and recollection. Based on the selective effects of SWS-related system consolidation (Diekelmann \& Born, 2010) on hippocampus-dependent associative memory, and thus potentially on recollection (Yonelinas, 2002), it is highly relevant to examine whether the consolidation effects related to REM sleep and SWS also differently modulate familiarity-based and recollection-based recognition. In accordance with prior findings, 
we expected item memory for emotional stimuli to be supported by late night REM sleep-rich sleep. Moreover, we aimed to test whether emotional source memory would benefit from early night SWS-rich sleep, as would be predicted based on the active system consolidation account (Diekelmann \& Born, 2010), or from late night REM sleep-rich sleep, consistent with findings on emotional item memory (Groch et al., 2013; Groch et al., 2015; Nishida et al., 2009; Wagner et al., 2001). In light of the literature referred to above (Chiu et al., 2013; Kensinger, 2009; Mather, 2007), we specifically designed our task procedure to establish conditions that should allow capturing potential effects of emotion on intrinsic feature encoding. We pursued this by manipulating a single perceptual source feature (location) and by promoting interactive imagery via specific encoding instructions (Bergstrom, Henson, Taylor, \& Simons, 2013). Thus, our procedure enforced deep engagement with the stimulus and its respective location, hence, enabling us to test the latter hypothesis under conditions which may render the source feature amenable to REM sleep-inherent emotional memory processing.

We further investigated the neural underpinnings of sleeprelated consolidation by analyzing theta power during REM sleep and spindle activity during SWS. Specifically, we aimed to explore how both oscillatory features are related to postsleep memory performance. In addition, we examined whether REM sleep has a simultaneous decremental effect on emotional reactivity of previously acquired memories (Walker \& van der Helm, 2009). To address this, we assessed subjective valence and arousal responses to emotional and neutral images following postsleep memory testing, which allowed us to contrast the effects of early night SWS-rich and late night REM sleep-rich sleep.

\section{Materials and methods}

\section{Experimental design}

The present study used a between-subjects split-night manipulation that yields a partial dissociation of the influences of REM sleep and SWS on processes of memory consolidation (Ekstrand, 1967; Groch et al., 2013; Groch et al., 2015; Menz, Rihm, \& Buchel, 2016; Plihal \& Born, 1997; Schreiner \& Rasch, 2015). Due to homeostatic demands building up over sustained wakefulness, long phases of SWS dominate the first night half with very short intervals of REM sleep within the ultradian sleep cycle. In the second night half, when the homeostatic pressure is dissipated, successively prolonged amounts of REM sleep appear with only minor co-occurring amounts of SWS. This distribution of sleep phases over the course of the night is reliably found across different individuals and can therefore be systematically manipulated by varying the time points of encoding and retrieval, respectively. In the current between-subjects design, one group was subjected to early night SWS-rich ("early sleep") sleep, whereas the second group underwent late night REM sleep-rich ("late sleep") sleep in the critical consolidation phase (see Fig. 1). The timing of the respective study and test phases was determined by the first stable sleep onset of the participant, with the 3-hour-interval starting at the first epoch of N1 (NREM Stage 1) succeeded by N2 (NREM Stage 2). This allows for a comparable dissipation of SWS over the first night half in all participants.

\section{Participants}

Forty-one subjects took part in the study. Participants were screened for neurological and psychiatric disorders, sleep disorders (Buysse, Reynolds, Monk, \& Hoch, 1991), habitual sleep duration ( $\geq 7$ hours), strong evening preference (Randler, 2013), alcohol and nicotine ingestion, regular shift work, and left-handedness (Oldfield, 1971). Additional requirements for study participation were normal or corrected-to-normal vision, normal weight $(18.5<\mathrm{BMI}<25)$ and no long-term use of medication. In order to exclude potentially confounding effects of menstrual cycle, only females under hormonal contraception for the preceding three months were included (Genzel et al., 2012). Participants were instructed to maintain a regular sleepwake pattern over the two weeks preceding the experimental night, which was monitored by a sleep diary, and to refrain from drinking alcohol or caffeinated beverages 24 hours before coming to the laboratory. Furthermore, subjects were asked to rise at 7:00 a.m. at the latest on the morning of the experiment. Prior to analyses, one participant was excluded due to meeting ICD-10 criteria of a moderate major depression, as assessed by the Patient Health Questionnaire (Kroenke, Spitzer, \& Williams, 2001). Data from two additional subjects were excluded (Tukey 1977) due to poor performance in source memory at posttest $(1.5 \times$ interquartile range $[\mathrm{IQR}]$ below the lower quartile of the overall group; $N=1$ ) and severe difficulties falling asleep (timing of posttest exceeded $1.5 \times$ IQR above the upper quartile of the late sleep group) leading to desynchronization in timing of postsleep procedures from the rest of the group $(N=$ 1). The final sample consisted of 19 participants in the early sleep group (six male, $M_{\text {age }}=22.9$ years, $S D_{\text {age }}=2.36$ ) and 19 subjects in the late sleep group (six male, $M_{\text {age }}=22.58$ years, $S D_{\text {age }}=2.24$ ). All participants gave written informed consent and were paid $100 €$ for complete study participation.

\section{Procedure}

All experimental procedures were performed at the Interdisciplinary Sleep Laboratory at Pfalzklinikum (Klingenmünster, Germany) on subsequent weekend days. Prior to the experimental night, participants underwent an adaption night in the sleep laboratory with complete electrode setup and under identical conditions as during experimental procedures. Subjects reported to the laboratory at 6:45 p.m. On 


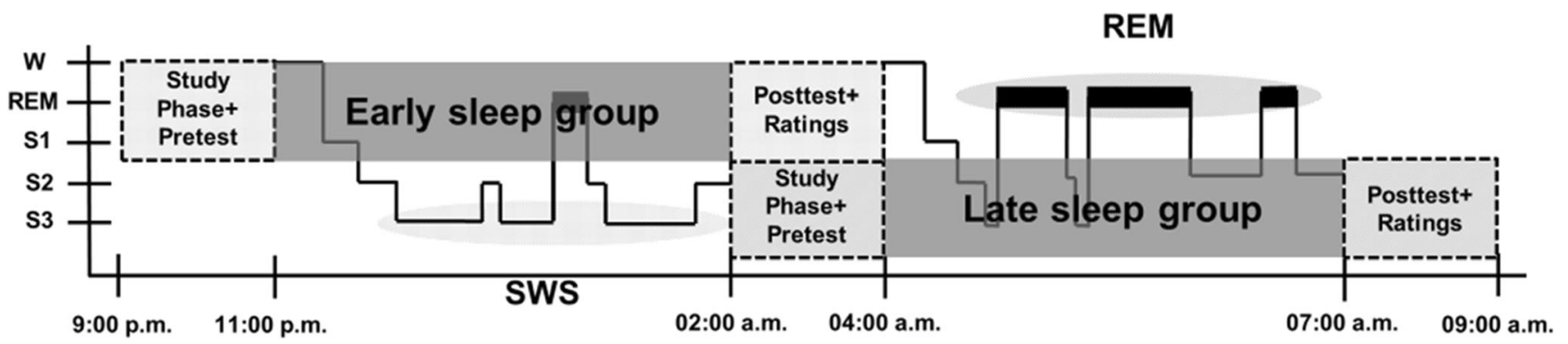

Fig. 1 Overview of the split-night design. Note. Participants of the early sleep group encoded images in the evening before sleeping and took the final postsleep retrieval test after sleep in the first night half. Participants of the late sleep group encoded images after the first night half and underwent postsleep testing after sleep in the second night half.
Consequently, both groups slept during the entire night, and only the timing of learning and test sessions was varied between conditions. Ratings refer to the emotional reactivity assessment that was timed following the posttest procedure arrival, they completed tests of fluid intelligence (Formann, Waldherr, \& Piswanger, 2011) as well as processing speed (Wechsler, 2008) and obtained an undisturbed sleep opportunity of eight hours. Participants went to bed at 11:00 p.m. and were woken up at 7:00 a.m., thus recreating the sleep interval of the main experiment.

During the experimental night, which took place one week after the adaption night, subjects arrived in the laboratory at 6:45 p.m. to undergo electrode setup and prepare for the night (see Fig. 1 for an overview of the study procedures). Allocation to study conditions was performed pseudorandomly in order to control for gender effects (Genzel et al., 2012). Due to practical reasons (preparation of individual testing rooms), participants were assigned to early and late sleep groups after the adaption night but prior to the main experiment. Thus, both experimenters and subjects remained blind to future assignment throughout the foregoing procedures. Participants of the early sleep group underwent encoding of the study material (starting at 9:00 p.m.) and a pretest followed by three hours of sleep in the first night half from 11:00 p.m. onwards, thus obtaining high amounts of SWS in the critical consolidation period. They underwent a final retrieval test as well as the emotional reactivity assessment at approximately 2:00 a.m. Participants of the late sleep group watched a neutral, nonnarrated documentary movie (excerpts of Samsara) without intentionally encoding the content before going to sleep at 11:00 p.m. for an identical sleep duration of three hours. Consequently, studying and pretesting took place following the first night half (approximately 2:00 a.m.), and postsleep memory performance and emotional reactivity were assessed in the morning at approximately 7:00 a.m. Hence, participants were tested after a consolidation period high in REM sleep. Prior to the respective study and posttest block, participants watched a 30-minute compilation of nonarousing film clips (taken from Relaxing: The Most Beautiful Landscapes on Earth) in order to prevent an effect of initial sleep inertia on memory performance. Before performing the encoding task as well as the final retrieval test, measures of state sleepiness (Hoddes, Zarcone, Smythe,
Phillips, \& Dement, 1973) and general mood (Watson, Clark, $\&$ Tellegen, 1988) were ascertained to account for potential circadian effects.

\section{Task design and sleep recordings}

\section{Stimulus material}

Pictorial stimuli for the memory task were selected from the International Affective Picture System (Lang, Bradley, \& Cuthbert, 2005) and supplemented with pictures from the Nencki Affective Picture System (Marchewka, Żurawski, Jednoróg, \& Grabowska, 2014) in order to allow for a sufficient number of trials. Negative pictures were preselected to be low in valence $(M=2.53, S D=0.66)$ and moderate to highly arousing $(M=6.25, S D=0.67)$, whereas neutral stimuli were chosen to be medium in valence $(M=5.5, S D=0.61)$ and moderate to low in arousal $(M=4.01, S D=0.81)$. To ascertain the applicability of the norms in the current student population, a prerating of the pictures with an unrelated sample of participants $(N=10)$ was conducted. Neutral and negative stimuli were closely matched in luminance, animacy, the occurrence of faces and indoor/outdoor displays. The final amount of 200 negative and 200 neutral items was split into four parallel sets equal in valence, arousal, and the aforementioned visual stimulus properties, which served as old and new items at pretest and posttest, respectively, and were counterbalanced across participants. Different sets of items were tested during pretest and posttest procedures in order to preclude effects of repeated testing, which may trigger rapid consolidation events (Antony, Ferreira, Norman, \& Wimber, 2017). To exclude the potential occurrence of primacy and recency effects and to allow adaption to task procedures three neutral and three negative pictures were presented prior to and following the target picture set. The order of stimulus presentation was pseudorandomized with no more than two stimuli of the same valence category occurring in succession and each participant receiving an individual order. 


\section{Memory Task}

During encoding, participants saw an initial fixation crosshair (200 ms), after which the stimulus was presented for 2,000 $\mathrm{ms}$. The source feature (screen location) was varied by presenting the stimulus on one of four different screen positions (right and left, as well as near or far from the screen center). After stimulus presentation, the participant's judgment of the encoding task ("Is the stimulus perceived as being positioned near or far from the screen center?") was ascertained $(2,000$ $\mathrm{ms}$ ) with the response keys counterbalanced across participants (see Fig. 2a). This encoding procedure was chosen in order to ensure interactive encoding of the source feature and the respective item (Bergstrom et al., 2013). Encoding was intentional with participants being instructed to memorize the items and their respective screen locations (right/left) for a subsequent test phase.
Prior to the pretest phase, which took place five minutes after encoding was completed, participants received standardized instructions for remember (R) and know (K) judgments (Rajaram, 1993). They were informed about the differences of both judgments, and the critical relevance of the explicit access to episodic details in distinguishing recollection from familiarity. They were instructed to give a "know" response when the image felt familiar in the absence of any recollective experience and to give a "remember" response in case they explicitly remembered contextual details from the study phase. Different examples were provided of episodic details, which could be retrieved in the current experimental setting (e.g., a specific thought that came to the participant's mind during initial image presentation or a particular sound coming from a different room that was heard by the participant during image presentation). It was emphasized that the a Encoding

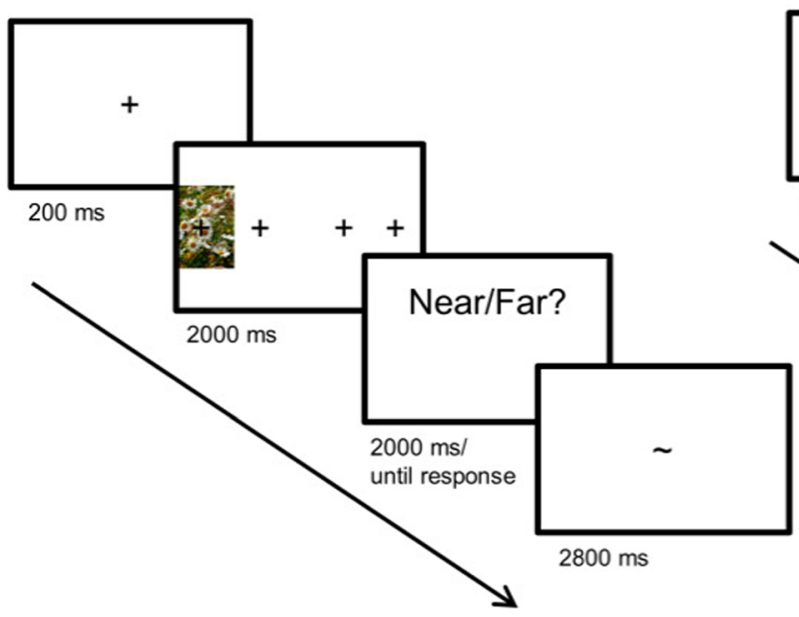

C Emotional Reactivity

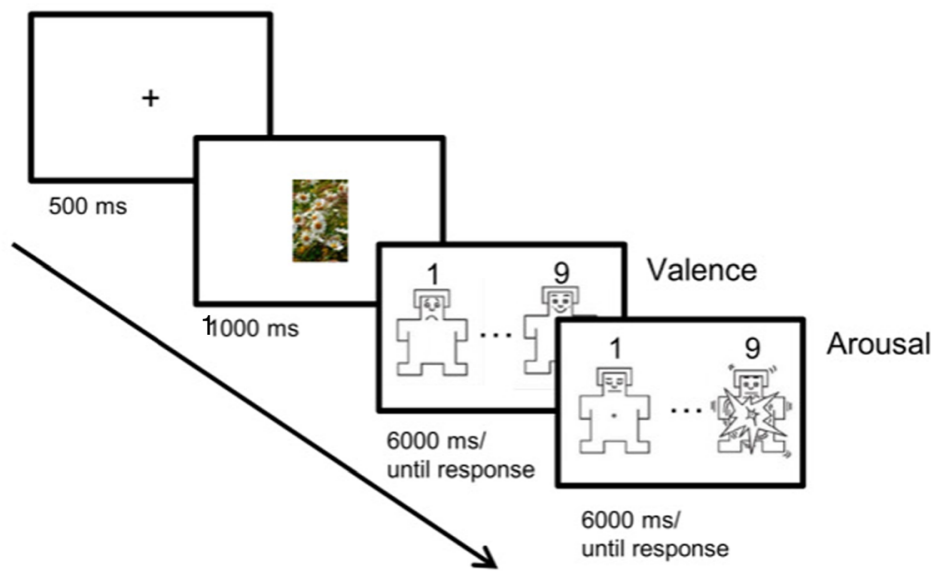

Fig. 2 Task design of (a) encoding procedure, (b) presleep and postsleep test procedure, and (c) emotional reactivity assessment 
emotionality of the stimulus itself was not relevant to this dissociation, but that a specific detail had to be retrieved in order to designate an image as "remembered." After receiving these instructions, participants performed a series of practice trials, in which they were asked to verbalize and justify their judgments to the instructor to ensure full comprehension. Each test trial began with the presentation of an initial fixation crosshair (200 $\mathrm{ms})$ that was followed by stimulus presentation $(1,500$ $\mathrm{ms}$ ) and an additional response window of $2,500 \mathrm{~ms}$ at stimulus offset (blank screen). Participants were instructed to respond as fast and as accurately as possible upon onset of stimulus presentation giving a remember/know/new judgment (see Fig. 2b). Response-key assignment was again counterbalanced across participants. For items that were indicated to be old ( $\mathrm{R}$ or $\mathrm{K}$ judgment), an additional prompt appeared (4,000 ms) asking participants to retrieve the initial screen location of the item (right/left/unknown). The procedures of the final postsleep retrieval test, during which the other half of the encoded items (50 neutral and 50 negative) was presented intermixed with an equal amount of new items, were kept identical to the pretest. The entire encoding procedure had an approximate duration of 45 minutes. The length of the retrieval tests varied between participants and lasted between 20 and 30 minutes.

\section{Emotional reactivity}

Emotional reactivity to previously encoded and new stimuli was assessed in a separate procedure in order to explore whether and how sleep affects the intensity of the emotional experience during item processing (see Fig. 2). Following the postsleep test, a separate picture-rating task was performed in which items of the posttest were presented in a different pseudorandomized order $(1,000 \mathrm{~ms})$. Instructions for the rating procedure were adapted from Lang et al. (2005), and individual ratings were ascertained on a standard 9-point selfassessment manikin scale (Bradley \& Lang, 1994). After initial presentation, the respective image remained on the screen while participants rated their subjective valence response $(6,000 \mathrm{~ms})$ by indicating whether they felt sad, unhappy, or melancholic (corresponding to a rating of 1) or happy, hopeful, pleased (corresponding to a rating of 9) while viewing the image (values 2-8 represented graded states between these extremes). After responding, a second scale was presented, asking participants to rate their subjective arousal response to the image $(6,000 \mathrm{~ms})$ by indicating whether they felt stimulated, excited, frenzied (corresponding to a rating of 9) or relaxed, calm, sluggish (corresponding to a rating of 1) while viewing the image (values 2-8 represented graded states between these extremes). Participants were instructed that given responses should reflect their immediate personal experience and what they actually felt during image presentation.

\section{Sleep recordings and spectral analysis}

During both sleep intervals, standard polysomnography recordings were performed according to the guidelines provided by the American Academy of Sleep Medicine (2007), including EEG at frontal, central, and occipital sites (F3, F4, C3, C4, O1, O2, according to the international 1020 system), EMG (submental) and horizontal EOG (lower right and higher left canthi). Signals were digitized at a sampling rate of $256 \mathrm{~Hz}$ (first-order high-pass and second-order Butterworth low-pass filter: 0.3 and $75 \mathrm{~Hz}$, respectively, notch filter: $50 \mathrm{~Hz}$ ) and amplified by SOMNOscreen amplifiers (SOMNOmedics GmbH, Randersacker, Germany). All electrodes were recorded referenced to $\mathrm{Cz}$ and were rereferenced off-line to the contralateral mastoid for sleepstage scoring and to the average of both mastoids for spectral analysis. A $0.3-35 \mathrm{~Hz}$ bandpass filter was applied off-line.

Visual sleep-stage scoring was performed by two trained raters independently in accordance with the criteria provided by the American Academy of Sleep Medicine (2007). The epoch length was set to $20 \mathrm{~s}$ to allow for overlapping windows of $4 \mathrm{~s}$ in the computation of spectral power density (see below). Each epoch was scored visually into NREM Stage N1, NREM Stage N2, NREM Stage N3 (corresponding to SWS), REM sleep (Stage R), and wake (Stage W). Both the absolute amount of minutes spent in each sleep stage as well as the relative amount with reference to total sleep time (\% TST) was determined for further analysis.

Spectral analysis was performed using the MATLABbased toolbox EEGLAB (http://www.sccn.ucsd.edu/eeglab/). Prior to analyses, epochs were rejected semi-automated on the basis of automatic detection of extremely large fluctuations $(>1000 \mu \mathrm{v})$ and thereafter applying a threshold of 5 standard deviations followed by visual identification of muscle and eye-movement artifacts. Spectral power density was computed for each epoch using the pwelch function (50\% overlap, Hamming window) with a resulting frequency resolution of 0 . $25 \mathrm{~Hz}$. Spectral power density was averaged for the theta band $(4.0-7.0 \mathrm{~Hz})$ during REM sleep and slow $(11.0-13.0 \mathrm{~Hz})$ as well as fast $(13.0-15.0 \mathrm{~Hz})$ spindle frequency ranges during SWS (Nuwer et al., 1998). For further analyses of theta power during REM sleep, the relative activity difference $\left(\mu v^{2} / \mathrm{Hz}\right)$ between left and right prefrontal sites was computed by subtraction of activity at electrode F3 from F4 (F4 - F3). To examine behavioral correlates of spindle density, mean absolute power $\left(\mu v^{2} / \mathrm{Hz}\right)$ in the frequency bands of interest is reported for frontal and central electrodes where amplitudes of sleep spindles have been found to be largest (Schabus et al., 2007). 


\section{Statistical analysis}

\section{Memory task}

Item memory performance was based on pictures correctly identified as new (correct rejections) and old (hits) collapsed across $\mathrm{R}$ and $\mathrm{K}$ judgments. To account for individual differences in response bias, corrected recognition scores $(\mathrm{Pr}$ index; Snodgrass \& Corwin, 1988) were calculated by subtracting the percentage of erroneous responses to new images (false alarms; FA) from the percentage of correctly recognized old images (hits). To further obtain an estimate of recollection-based recognition, the proportion of new items erroneously receiving an $\mathrm{R}$ judgment was subtracted from the proportion of old items correctly receiving an $\mathrm{R}$ judgment $\left(\mathrm{R}=\mathrm{R}_{\text {hits }}-\mathrm{R}_{\mathrm{FA}}\right)$. As the probability of making a $\mathrm{K}$ judgment is constrained by $\mathrm{R}$ judgments (participants are instructed to make a $\mathrm{K}$ judgment in the absence of recollection when the item is perceived as being familiar), an estimate of familiarity was derived $\left[\mathrm{K}=\mathrm{K}_{\text {hits }} /\left(1-\mathrm{R}_{\text {hits }}\right)-\mathrm{K}_{\mathrm{FA}} /\right.$ $\left(1-\mathrm{R}_{\mathrm{FA}}\right)$ ] according to Yonelinas and Jacoby (1994). Source memory performance was calculated by dividing the probability of a correct source judgment by the probability of a correct "old" response $\left(\mathrm{SM}=\mathrm{p}_{\text {correct source }} / \mathrm{p}_{\text {correct }}\right.$ source + incorrect source). As the current study design relied on between-subjects comparisons (for former within-subjects approaches, see, e.g., Groch et al., 2013; Groch et al., 2015), behavorial data were analyzed by means of factorial ANCOVAS to account for unrelated variance in memory performance (Miller \& Chapman, 2001). Preexperimentally ascertained measures of fluid intelligence (Formann et al., 2011) and processing speed (Wechsler, 2008) were introduced as covariates, as these are known to be highly influential with regard to memory performance (for fluid intelligence, see, e.g., Unsworth, Fukuda, Awh, \& Vogel, 2014; for processing fleuncy, see, e.g., Park et al., 1996). Response accuracy data were subjected to analyses of covariance (ANCOVA), including the within-subjects factors of time (pretest/posttest) and emotion (negative/neutral) as well as the between-subjects factor group (early/late sleep group). Mean reaction times for hits and correct rejections of the item memory test were analyzed in an ANCOVA, including the additional within-subjects factor of response type (hits/correct rejections).

\section{Emotional reactivity}

Emotional reactivity measures were seperately aggregated to mean arousal and valence ratings for hits and correct rejections of the preceding item memory procedure. This was done to directly compare reactivity to emotional and neutral items that had undergone sleep-related processing (hits) to that of images that were newly presented after sleep (correct rejections). Mean ratings were analyzed in separate ANOVAs, including the within-subjects factors emotion (negative/neutral), response type (hits/correct rejections), and the between-subjects factor group (early/late sleep group).

\section{Sleep physiology data}

To explore the relationship between memory performance and sleep electrophysiological data, Pearson correlation coefficients were computed between item/source memory and time spent in REM sleep, SWS, and total sleep time ${ }^{1}$ as well as spectral power density during specific sleep stages. To allow for a stronger dissociation between effects of sleep on item and source memory, partial correlations are reported controlling for shared variance whenever appropriate.

During sleep recordings, electrodes F3 ( $N=1$ participants; early sleep group) and $\mathrm{C} 4(N=2$ participants; late sleep group) lost sufficient skin contact and respective power values were substituted (means of surrounding electrodes), with the exception of frontal laterality in theta power (F4 - F3). Memory performance measures were adjusted when participants indicated to have erroneously pressed keys in specific trials according to item status ( $N=5$ trials across all participants).

Significant main effects and interactions were followed by a priori specified post hoc $t$ tests. In all post hoc and correlational analyses of memory performance, the Benjamini and Hochberg (1995) procedure for controlling false discovery rate (FDR) was used to take into account multiple comparisons with an FDR of 5\%. The procedure was not applied to specific exploratory follow-up analyses, ${ }^{2}$ as these only serve to illustrate effects that previously underwent FDRcontrolled analyses. For explorative analyses on arousal and valence ratings, uncorrected $p$ values are also reported.

\section{Results}

\section{Behavioral data}

\section{A priori group comparisons}

Presleep item memory performance was analyzed in separate ANOVAs, involving the factors group and emotion. There was neither a main effect of group, $\mathrm{F}(1,36)=0.20, p=.658$, nor a Group $\times$ Emotion interaction, $F(1,36)=0.38, p=.539$. In a similar vein, source memory performance prior to sleep was not modulated by the group factor, group: $F(1,36)=1.79, p=$

\footnotetext{
${ }^{1}$ To differentiate potential effects of respective sleep stages from effects of general sleep duration.

${ }^{2}$ See subanalyses in each frequency bin in the theta frequency range (see Fig. 6) and the correlation between fast spindle power and the relative retention benefit of neutral relative to emotional item memory (see Fig. 7b).
} 
.189 ; Group $\times$ Emotion: $F(1,36)=1.58, p=.218$. Participants' accuracy in the encoding task (correctly indicating the image location immediately after its presentation) was generally very high, as the means of both groups approached full performance rates at 1.0. Also, groups did not differ significantly in their performance on the encoding task, $t(36)=1.22, p=.230$. Groups were also closely comparable in covariate measures of fluid intelligence, $t(36)=0.39, p=.697$, and processing speed, $t(36)=0.93, p=.359$. To examine potential contributions of circadian effects, ratio of positive to negative mood (Diener, 2000) was compared at pretest (prior to encoding) and posttest (before the final retrieval test) between both groups (see Table 1). Mood ratio was similar between groups across both time points, pre: $t(36)=0.91, p=.368$; post: $t(36)=0.40, p=.690$, as was subjectively reported sleepiness at encoding, $t(36)=0.47, p=.641$. Before postsleep memory testing participants in the early sleep group reported elevated levels of sleepiness as compared to the late sleep group, $t(36)=5.00, p<.001$ (see Groch et al., 2015, for similar findings). However, none of the postsleep memory measures were significantly correlated with this subjective level of sleepiness (highest correlation: $r=.14, p=.400$ with neutral source memory performance), rendering this an unlikely cause of differential group patterns.

\section{Item memory}

Analysis of item memory revealed significant main effects of time, $F(1,34)=156.43, p<.001$, and emotion, $F(1,34)=$ $13.88, p=.001$, as well as a significant interaction between both factors, $F(1,34)=9.24, p=.005$, pointing to an enhanced retention of emotional stimuli from pretest to posttest (see Fig. 3a). Neutral item memory exhibited a stronger decline over time, $F(1,35)=123.11, p<.001, \omega^{2}=.77$, as compared to emotional item memory, $F(1,35)=34.31, p<$ $.001, \omega^{2}=.47$. Consequently, while there was no significant difference in initial memory performance between the two valence categories at pretest, $F(1,35)=2.48, p=.124$, a significant difference emerged over time, as evident at posttest, $F(1,35)=19.5, p<.001$. No significant main effect, $F(1$, $34)=1.84, p=.184$, or interactions involving the group factor, Time $\times$ Group: $F(1,34)=0.08, p=.776$; Emotion $\times$ Group: $F(1,34)=1.48, p=.233$; Time $\times$ Emotion $\times$ Group: $F(1,34)=$ $0.84, p=.366$, were found with regard to item memory performance, suggesting that this memory benefit was not modulated by the early/late sleep manipulation (see Table 2 for descriptive statistics).

Analysis of reaction times (ms) of hits and correct rejections of the item memory test revealed significant main effects of emotion, $F(1,34)=80.42, p<.001$, and response type, $F(1,34)=130.74, p<.001$, as well as a significant Emotion $\times$ Response Type interaction, $F(1,34)=78.15, p<.001$. Post hoc tests indicated that participants were generally faster when making correct "new" judgments as compared to correct "old" judgments, $F(1,35)=$ $125.16, p<.001$. Additionally, subjects were significantly faster when responding correctly to new items when the stimulus was emotional, $F(1,35)=129.63, p<.001$ (see Supplementary Data 1). Again, no main effect of group emerged in these analyses, $F(1,34)=2.15, p=.152$, as well as no interaction involving the group factor, Time $\times$ Group: $F(1,34)=0.06, p=.809$; Emotion $\times$ Group: $F(1$, $34)=0.04, p=.843$; Response Type $\times$ Group: $F(1,34)=$ $2.56, p=.119$; Time $\times$ Emotion $\times$ Group: $F(1,34)=1.79$, $p=.190$; Time $\times$ Response Type $\times$ Group: $F(1,34)=$ $0.20, p=.658$; Emotion $\times$ Response Type $\times$ Group: $F(1$, 34) $=0.01, p=.937$; Time $\times$ Emotion $\times$ Response Type $\times$ Group: $F(1,34)=0.01, p=.934$.

Table 1 Descriptive group measures

\begin{tabular}{llrl}
\hline Measure & Early sleep group $(N=19)$ & Late sleep group $(N=19)$ & Test statistics \\
\hline WMT-2 & $13.05(.66)$ & $13.42(.67)$ & $\mathrm{t}(36)=0.39, p=.697$ \\
DSST & $65.16(2.02)$ & $68.00(2.29)$ & $t(36)=0.93, p=.359$ \\
Accuracy encoding task & $.98(.04)$ & $.99(.01)$ & $\mathrm{t}(36)=1.22, p=.230$ \\
SSS encoding & $2.84(0.96)$ & $3.00(1.11)$ & $t(36)=0.47, p=.641$ \\
SSS posttest & $4.11(1.29)$ & $2.32(0.89)$ & $t(36)=5.00, p<.001$ \\
PANAS encoding & $1.04(0.20)$ & $1.09(0.15)$ & $t(36)=0.91, p=.368$ \\
PANAS posttest & $1.10(0.13)$ & $1.08(0.17)$ & $t(36)=0.40, p=.690$ \\
\hline
\end{tabular}

Note. WMT-2 = Wiener Matritzen Test-2 [Viennese Matrices Test-2]; DSST = Digit Symbol Substitution Test; Accuracy in performing the encoding task is expressed as the percentage of correct responses (1 corresponding to maximal performance); SSS = Stanford Sleepiness Scale (single item measure ranging from 1 = feeling active, vital, alert, or wide awake to 7 = no longer fighting sleep, sleep onset soon; having dream-like thoughts); PANAS = Positive and Negative Affect Schedule - presented means are derived by computing the ratio of positive (positive affect subscale) to negative (negative affect subscale) mood (see Diener, 2000, for the reasoning behind this ratio and its advantages toward a disjunctive analysis of both subscales), a ratio of 1 indicates approximately balanced positive and negative mood, values $>1$ indicate a relative dominance of positive mood, values $<1$ indicate a dominance of negative mood, positive and negative subscales each comprise 10 adjectives related to current mood state, which are scored with regard to their current dominance on a scale from $1=$ not at all to $5=$ extremely). Standard deviations are given in parentheses 


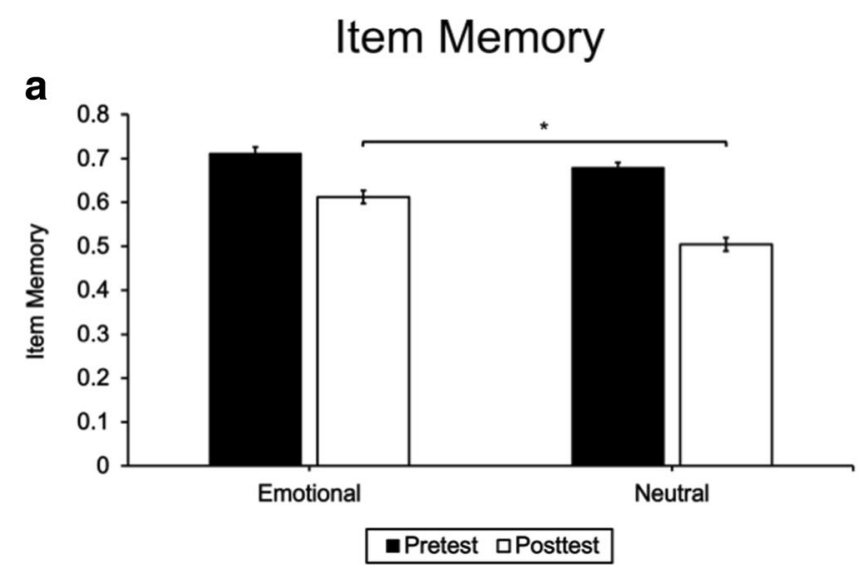

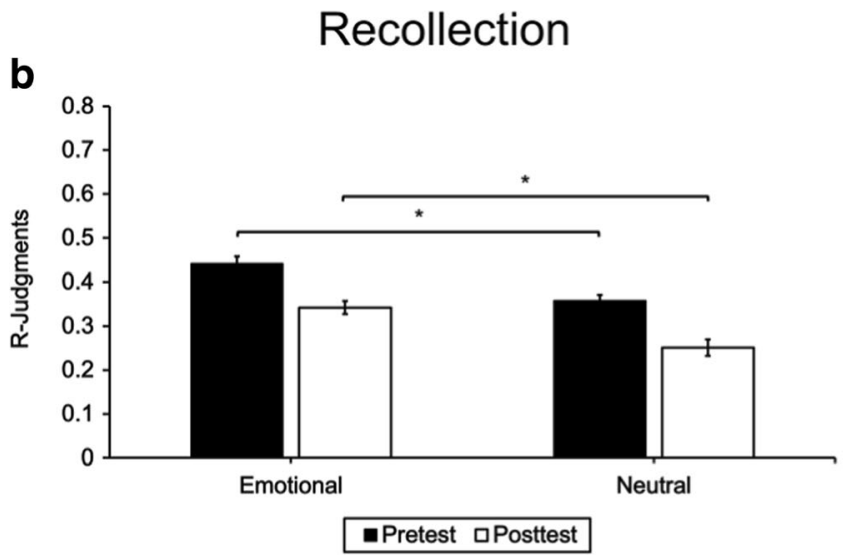

Fig. 3 Adjusted means of (a) item memory performance for emotional and neutral images and of $(\mathbf{b}+\mathbf{c}) \mathrm{R}$ and $\mathrm{K}$ judgments at pretest and posttest. Note. Performance rates are collapsed across groups as there were no significant main or interaction effects involving group. Adjusted means were computed by means of multiple regression controlling for mean-centered scores of intelligence and processing

\section{Remember and know judgments}

To further elucidate how this consolidation benefit for emotional images was reflected in recollection and familiarity measures separate ANCOVAs were performed (see Fig. 3b-c). Analysis of $\mathrm{R}$ judgments revealed significant main effects of time, $F(1,34)=31.98, p<.001$, and emotion, $F(1,34)=$ $20.85, p<.001$, indicating that $\mathrm{R}$ judgments were more frequent at pretest than at posttest and that emotional images were more likely to evoke $\mathrm{R}$ judgments than neutral images, irrespective of time and sleep, group: $F(1,34)=$ $1.44, p=.238$; Group $\times$ Time: $F(1,34)=0.90, p=.351$; Group $\times$ Emotion: $F(1,34)=0.12, p=.733$; Group $\times$ Emotion $\times$ Time: $F(1,34)=0.02, p=.893$.

$\mathrm{K}$ judgments were also modulated by time, $F(1,34)=$ $38.23, p<.001$, and emotion, $F(1,34)=16.96, p<.001$. Furthermore, a marginally significant Time $\times$ Emotion interaction, $F(1,34)=4.09, p=.051$, was apparent, indicating a differential decline of familiarity-based recognition for emotional and neutral items across time, as reflected by a
Familiarity

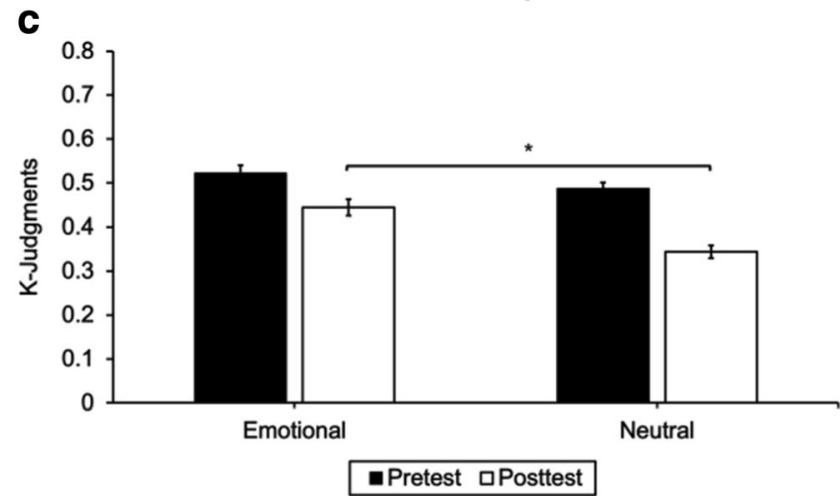

speed. $* p=.05$ for comparisons between valence categories at pretest and posttest. Benjamini-Hochberg correction was applied, allowing for $5 \%$ false positives. Significant effects over time are not illustrated for reasons of comprehensibility. Error bars represent the standard error following Cousineau-Morey corrections for within-subject designs (Cousineau, 2005; O’Brien \& Cousineau, 2014)

significant difference in the retention rates of emotional and neutral items after sleeping, $F(1,35)=17.43, p<$ .001 , but not before sleep, $F(1,35)=2.29, p=.140$. No main effect of group, $F(1,34)=0.30, p=.591$, or interaction effect involving the group factor, Group $\times$ Time: $F(1,34)=$ $0.02, p=.895$; Group $\times$ Emotion: $F(1,34)=3.13, p=.086$; Group $\times$ Emotion $\times$ Time: $F(1,34)=0.35, p=.559$, was evident in these analyses. Consequently, the retention benefit for emotional stimuli observed with regard to item memory (see Fig. 3a) mainly resulted from preserved familiarity-based recognition for these items at posttest (see Fig. 3c; see also $\mathrm{Hu}$ et al., 2006, for similar findings).

\section{Source memory}

Similar to item memory, source memory performance declined from pretest to posttest, time: $F(1,34)=17.58, p<.001$. No main effect of group, $F(1,34)=1.30, p=.262$, emerged in these analyses, and no significant interaction was attained between group and time, $F(1,34)=0.43, p=.519$, nor between group 
Table 2 Adjusted means of memory performance in both groups for item and source memory

\begin{tabular}{|c|c|c|c|c|c|}
\hline \multirow[t]{2}{*}{ Memory test } & \multirow[t]{2}{*}{ Performance measure } & \multicolumn{2}{|c|}{ Early sleep group $(N=19)$} & \multicolumn{2}{|c|}{ Late sleep group $(N=19)$} \\
\hline & & Pretest & Posttest & Pretest & Posttest \\
\hline \multirow[t]{6}{*}{ Item memory } & $\mathrm{PR}_{\text {Emo }}$ & $.72(.03)$ & $.61(.03)$ & $.70(.03)$ & $.62(.03)$ \\
\hline & $\mathrm{PR}_{\text {Neut }}$ & $.70(.03)$ & $.53(.04)$ & $.66(.03)$ & $.48(.04)$ \\
\hline & Hits $_{\text {Emo }}$ & $.84(.02)$ & $.75(.03)$ & $.79(.02)$ & $.73(.03)$ \\
\hline & Hits $_{\text {Neut }}$ & $.76(.03)$ & $.61(.04)$ & $.70(.03)$ & $.56(.04)$ \\
\hline & $\mathrm{FA}_{\text {Emo }}$ & $.12(.03)$ & $.14(.02)$ & $.09(.03)$ & $.12(.02)$ \\
\hline & $\mathrm{FA}_{\text {Neut }}$ & $.06(.01)$ & $.07(.02)$ & $.04(.01)$ & $.08(.02)$ \\
\hline \multirow[t]{4}{*}{ RK procedure } & $\mathrm{R}_{\text {Emo }}$ & $.48(.05)$ & $.37(.04)$ & $.40(.05)$ & $.32(.04)$ \\
\hline & $\mathrm{R}_{\text {Neut }}$ & $.41(.05)$ & $.28(.04)$ & $.31(.05)$ & $.22(.04)$ \\
\hline & $\mathrm{K}_{\mathrm{Emo}}$ & $.50(.04)$ & $.41(.05)$ & $.55(.04)$ & $.48(.05)$ \\
\hline & $\mathrm{K}_{\text {Neut }}$ & $.48(.05)$ & $.35(.04)$ & $.49(.05)$ & $.34(.04)$ \\
\hline \multirow[t]{2}{*}{ Source memory } & $\mathrm{SM}_{\mathrm{Emo}}$ & $.59(.03)$ & $.53(.03)$ & $.62(.03)$ & $.58(.03)$ \\
\hline & $\mathrm{SM}_{\text {Neut }}$ & $.58(.03)$ & $.55(.04)$ & $.66(.03)$ & $.56(.04)$ \\
\hline
\end{tabular}

Note. Adjusted means were computed by means of multiple regression controlling for mean-centered scores of intelligence and processing speed. $\mathrm{PR}=$ Hits-FA; Hits = correctly recognized images of the item memory procedure; $\mathrm{FA}=$ false alarms to new images of the item memory procedure; $\mathrm{R}=$ estimate of recollection derived from the amount of correct remember judgments of the item memory procedure adjusted by the rate of false alarms; $\mathrm{K}=$ estimate of familiarity derived from the amount of correct know judgments of the item memory procedure adjusted by the rate of false alarms; SM = correctly recognized locations of items that had been correctly recognized in the preceding item memory procedure (correct source/overall hits). Standard errors of the mean are given in parentheses. None of the reported mean values reached the significance level in between-group comparisons $(p>.05)$

and emotion, $F(1,34)=0.01, p=.991$. Notably, however, a significant three-way Time $\times$ Emotion $\times$ Group interaction emerged, $F(1,34)=5.2, p=.029 .{ }^{3}$ Planned comparisons between groups did not reveal any significant differences in source memory after sleep for either neutral, $F(1,34)=0.04, p=.838$, or emotional images, $F(1,34)=1.93, p=.174$. Group-specific analyses, however, revealed that source memory performance for emotional items declined significantly from pretest to posttest in the early sleep group, $F(1,16)=8.24, p=.011$, whereas for neutral source memory, no significant decline, $F(1,16)=2.43, p$ $=.138$, was evident. On the other hand, in the late sleep group, source memory for neutral stimuli was found to decrease significantly, $F(1,16)=14.67, p=.001$, but remained preserved for emotional stimuli, $F(1,16)=3.00, p=.103$ (see Fig. 4).

By showing selectively enhanced retention of neutral source memory in the early sleep group, these results support the high relevance of SWS for the consolidation of hippocampusdependent memory representations. Similar effects of SWS on source memory have recently been shown to be limited to neutral images (Groch et al., 2015). By showing that REM sleep, on the other hand, has the capacity to selectively support source memory for emotional stimuli, the current results extend prior findings in an important way. Furthermore, these results also imply that emotional source memory benefits from a sleeprelated consolidation mechanism that is different from the one mediating the consolidation of neutral stimuli.

\footnotetext{
${ }^{3}$ The three-way interaction remained significant without controlling for IQ and processing speed, $F(1,36)=4.52, p=.04$.
}

\section{Emotional reactivity}

In order to examine potential simultaneous effects of REM sleep on emotional responses towards experimental stimuli, valence and arousal ratings were subjected to separate ANOVAs. As expected, valence ratings differed significantly between valence categories, emotion: $F(1,36)=354.24, p<$ .001 , with negative images eliciting lower valence ratings than neutral images. In addition, a significant Emotion $\times$ Response Type (hit/correct rejection) interaction emerged, $F(1,36)=$ $5.29, p=.027$. The difference in ratings between new items (correct rejections) and old items (hits) was significantly larger for emotional images as opposed to difference values for neutral pictures, $t(37)=2.29, p=.028$. In other words, in the emotional valence category "old" images that had been initially presented prior to sleeping in the respective night half tended to be perceived as more aversive as compared to "new" images, which were presented only during the posttest after sleeping, $t(37)=1.93, p=.061$ (see Fig. 5a). Analysis on valence ratings did not yield any significant main or interaction effects involving the group factor, group: $F(1,36)=1.39$, $p=.246$; Group $\times$ Emotion: $F(1,36)=3.46, p=.071$; Group $\times$ Response Type: $F(1,36)=0.18, p=.671$; Group $\times$ Emotion $\times$ Response Type: $F(1,36)=1.26, p=.269$.

Analysis on arousal ratings also revealed the expected significant main effect of emotion, $F(1,36)=267.20, p<.001$, signifying a markedly enhanced arousal response to emotional as opposed to neutral stimuli. Furthermore, it was found that old items were perceived as more arousing in comparison to new 


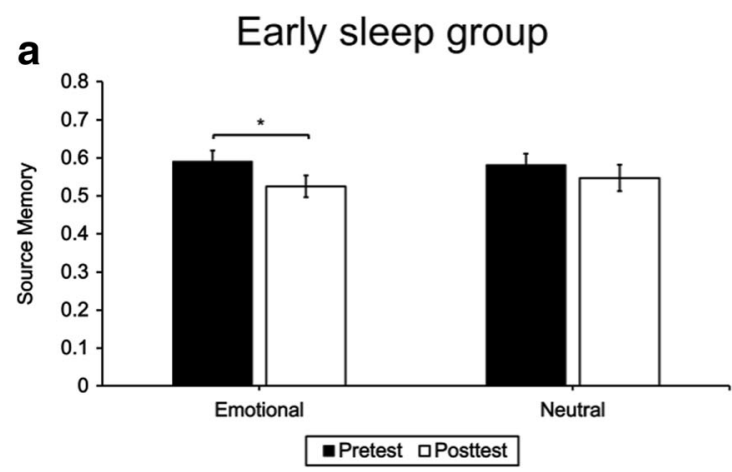

Fig. 4 Adjusted means of source memory performance (ratio of correct source judgments to item memory hits) for emotional and neutral items in the (a) early and (b) late sleep group at pretest and posttest. Note. Withingroup effects of differential memory retention in relation to valence category were qualified by a significant three-way-interaction of Group $\times$ Time $\times$ Emotion, $F(1,34)=5.2, p=.029$. Adjusted means were

items across both valence categories, response type: $F(1,36)=$ $7.36, p=.01$. Additionally, a significant Emotion $\times$ Group interaction was apparent, $F(1,36)=5.01, p=.032 .{ }^{4}$ Although there was no significant difference in arousal responses to neutral stimuli between both groups, $t(36)=0.72, p=.477$, participants in the late sleep group indicated to perceive emotional stimuli as significantly more arousing when compared to subjects in the early sleep group, irrespective of response type, $t(36)=2.11, p=.042$ (see Fig. 5b).

Thus, contrary to our hypothesis that REM sleep has an attenuating effect on the intensity of emotional experiences elicited by reprocessed material, REM sleep was found to be associated with an enhanced arousal response to emotional stimuli. This was evident irrespective of whether these images had been initially presented prior to (hits) or following (correct rejections) sleep, which precludes that this effect results from memory processes during the consolidation period. No further main or interaction effects involving the group factor emerged in these analyses, group: $F(1,36)=3.14, p=.085$; Group $\times$ Response Type: $F(1,36)=0.06, p=.804$; Group $\times$ Emotion $\times$ Response Type: $F(1,36)=1.21, p=.280$.

\section{Sleep physiology: sleep stages}

Data on absolute and relative time spent in different sleep stages (in min, \% TST) is presented in Table 3. The critical manipulation of the split-night design was confirmed by demonstrating highly significant differences in REM sleep, $t(36)=$ $12.32, p<.001$, and N3/SWS, $t(36)=9.12, p<.001$, duration between the early and late sleep groups in the expected

\footnotetext{
${ }^{4}$ Additional analyses contrasting responses to all new and old images (rather than correctly recognized old and new images) of the posttest procedure similarly yielded a significant Group $\times$ Emotion interaction, $F(1,36)=5.15, p=$ .029 , in the absence of any further group-related effects. Corresponding analysis of valence ratings did not yield any group-related effects.
}

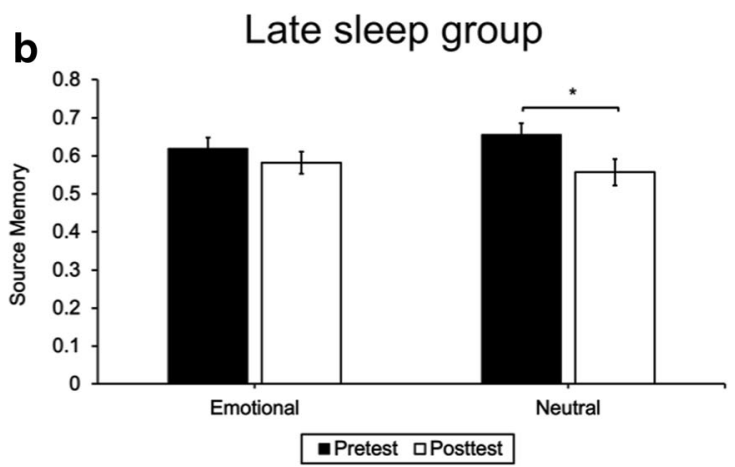

computed by means of multiple regression controlling for meancentered scores of intelligence and processing speed. ${ }^{*} p=.05$ for comparisons within valence categories from pretest to posttest. Benjamini-Hochberg correction was applied allowing for 5\% false positives. Error bars represent the standard error

direction. Importantly, both groups did not differ significantly with respect to time spent in any other sleep stage and exhibited a similar total sleep time, $t(36)=1.26, p=.215$.

Partial correlations between sleep physiology data and memory performance, controlling for shared variance between item and source memory, were computed separately for both groups. Contradicting prior findings (Groch et al., 2013; Nishida et al., 2009), there was no significant correlation between time spent in REM sleep and emotional postsleep memory performance in the early (source memory: $r=.02, p=.923$; item memory: $r=.16, p=.516$ ) and late sleep groups (source memory: $r=.02, p=.927$; item memory: $r=.07, p=.772$ ), respectively. However, the procedures of the split-night design result in a very narrow range in total sleep time. Consequently, variance between subjects regarding time spent in different sleep stages within each condition is low, which may have prevented finding the respective correlations (see Groch et al., 2015, for similar results).

In a similar vein, correlational analyses between mean arousal and valence ratings of emotional images and measures of sleep physiology did not yield any significant associations with REM sleep duration. However, a differential association emerged between total sleep time and arousal ratings of emotional stimuli in the early group $(r=-.54, p=.016)$, which was not evident in the late sleep group $(r=.21, p=.390)$. A full account of correlational results and a more detailed discussion of exploratory findings with regard to total sleep time can be found in the supplementary materials (see Supplementary Data 2).

\section{Sleep physiology: spectral analysis}

To test whether microstructural aspects of REM sleep physiology would be associated with behavioral memory performance, correlations between memory measures and rightfrontal theta lateralization (F4 - F3) were analyzed based on 


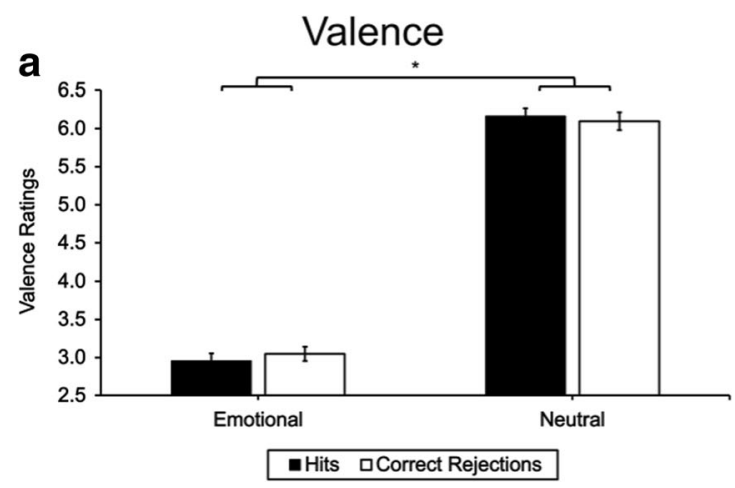

Fig. 5 Mean postsleep ratings of stimulus material separately for emotional and neutral items on (a) valence and (b) arousal dimensions. Note. Valence and arousal were rated on a 9-point-scale. High valence values reflect positive valence, and low valence values reflect negative valence. To depict significant interactions, valence ratings are collapsed across groups and depicted separately for response types (hits/CR),

the findings of Nishida et al. (2009). The correlational patterns mirrored behavioral results, by demonstrating no significant association between frontal theta lateralization and emotional item memory performance $(r=-.36, p=.146)$. This is in line with the absence of any particular effect of late REM sleeprich sleep on emotional item memory performance. However, in the late sleep group, there was a significant positive correlation between right-frontal theta lateralization and postsleep source memory for emotional images $(r=.55, p=.018)$. Critically, this was not evident for neutral source memory $(r$ $=.23, p=.369$ ). Moreover, the correlations between emotional and neutral source memory performance were confirmed to differ significantly on a statistical level (Fisher's $Z_{r}=2.17, p=$ $\left..015^{5}\right)$. A more fine-grained analysis within each $0.25 \mathrm{~Hz}$ bin (see Fig. 6a) in the theta frequency range revealed the largest correlation in the $4.75-5 \mathrm{~Hz}$ bin $(r=.59, p=.011)$ and generally stronger correlations in the lower $(<5 \mathrm{~Hz})$ frequency range (see Fig. 6b). Analysis in the early sleep group did not yield any significant correlations between source (emotional: $r$ $=.19, p=.470$; neutral: $r=.14, p=.607$ ) or item memory performance (emotional: $r=-.40, p=.109$; neutral: $r=-.04$, $p=.866$ ) and right-frontal theta lateralization. Thus, consistent with our behavioral findings, disclosing a selective retention benefit for emotional source memory after late REM sleeprich sleep, we were additionally able to identify an electrophysiological process, right-frontal theta lateralization, which is specifically bound to the enhanced retention of emotional source memory.

Based on the predictions of the active system consolidation view, we further tested whether memory performance for neutral items would be correlated with spindle power during SWS. The analysis of fast $(13-15 \mathrm{~Hz})$ and slow $(11-13 \mathrm{~Hz})$

\footnotetext{
$\overline{5}$ One-sided test based on the procedure described by Eid, Gollwitzer, and Schmitt (2010).
}

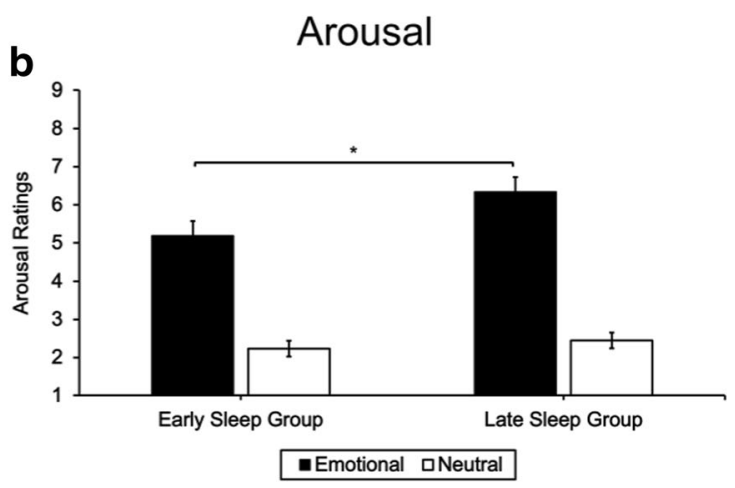

whereas arousal ratings are collapsed across response types and depicted separately for early and late sleep groups. $* p=.05$ without control of false discovery rate. Error bars represent the standard error. For valence ratings, standard errors were calculated following Cousineau-Morey corrections for within-subject designs (Cousineau, 2005; O'Brien \& Cousineau, 2014)

spindle frequency ranges in the early sleep group revealed no significant correlation with postsleep source memory performance (see Table 4). However, postsleep neutral item memory performance was found to correlate significantly with slow spindle power at all electrodes and with fast spindle power at electrode F3 (see Fig. 7a). This was not true for emotional item memory, providing further evidence for a selective role of SWS in memory consolidation for neutral items.

This differential pattern with regard to stimulus valence was again confirmed by statistical analyses, directly contrasting the strength of correlations with emotional and neutral item memory for fast spindle power (F3) and slow spindle power at all electrodes (see caption of Table 4 for detailed statistics). Interestingly, frontal fast spindle power (F3) also demonstrated a particularly high correlation with memory performance for neutral relative to emotional images $(r=.69, p=$ $.001){ }^{6}$ This association signifies that higher spectral power in the fast spindle band $(13-15 \mathrm{~Hz})$ during SWS was associated with a greater retention benefit of neutral over emotional item memory at posttest (see Fig. 7b). No significant correlations were found in the late sleep group (see Table 4).

In sum, we were able to identify dissociable neural correlates for the retention of neutral and emotional memories in both night halves. The selective retention benefit for emotional source memory in the late sleep group was correlated with right-frontal theta lateralization, whereas spindle power, a key feature of SWS, was selectively correlated with neutral item memory in the early sleep group. This pattern does not fully conform to the behavioral dissociation, which manifested itself specifically in source memory performance decrements across the first and second night halves. Nonetheless, the present neurophysiological findings importantly corroborate that

\footnotetext{
${ }^{6}$ PrNeut $_{\text {post - PrEmo }}{ }_{\text {post }}$; see Prehn-Kristensen et al. (2013) for a similar approach in data analysis.
} 
Table 3 Sleep parameters in both study groups

\begin{tabular}{|c|c|c|c|c|c|c|}
\hline \multirow[b]{2}{*}{ Sleep parameters } & \multicolumn{2}{|c|}{ Early sleep group $(N=19)$} & \multicolumn{2}{|c|}{ Late sleep group $(N=19)$} & \multicolumn{2}{|l|}{ Test statistics } \\
\hline & $\%$ TST & Time in $\min$ & $\%$ TST & Time in $\min$ & $\%$ TST & Time in min \\
\hline W & $1.74(2.69)$ & $3.11(4.67)$ & $0.97(1.65)$ & $1.78(3.06)$ & $t(36)=1.06, p=.295$ & $t(36)=1.04, p=.308$ \\
\hline N1 & $4.91(2.42)$ & $9.00(4.42)$ & $5.96(3.31)$ & $11.15(6.08)$ & $t(36)=1.12, p=.271$ & $t(36)=1.25, p=.221$ \\
\hline $\mathrm{N} 2$ & $40.78(8.29)$ & $75(15.47)$ & $44.29(8.51)$ & $82.55(15.61)$ & $t(36)=1.29, p=.206$ & $t(36)=1.50, p=.143$ \\
\hline $\mathrm{N} 3$ & $43.58(10.68)$ & $80.04(19.3)$ & $16.3(7.49)$ & $30.44(14.14)$ & $t(36)=9.12, p<.001$ & $t(36)=9.04, p<.001$ \\
\hline $\mathrm{R}$ & $10.73(6.88)$ & $19.87(13.03)$ & $33.44(4.15)$ & $62.49(8.51)$ & $t(36)=12.32, p<.001$ & $t(36)=11.94, p<.001$ \\
\hline TST & - & $183.91(6.77)$ & - & $186.63(6.48)$ & - & $t(36)=1.26, p=.215$ \\
\hline
\end{tabular}

Note. $\mathrm{W}=$ wake; $\mathrm{N} 1$ = NREM Stage $1 ; \mathrm{N} 2=$ NREM Stage 2; N3 = NREM Stage 3 (corresponding to SWS); R = REM sleep; TST = total sleep time. Standard deviations are given in parentheses

memory processes of both night halves are selectively involved in the processing of neutral and emotional material, respectively.

\section{Experiment 2: effects of wakeful consolidation}

In order to address alternative hypotheses for the behavioral effects of our main experiment, we conducted an additional experiment (Experiment 2) investigating consolidation in the same task design across a phase of wakefulness during the daytime. Regarding the interaction effect of time and emotion on item memory performance, our main study design does not allow to infer whether this retention benefit of emotional images was effected specifically across sleep-related consolidation. Alternatively, it may be argued that this reflects generic processes of consolidation, in the sense that emotional items show a benefit in retention over time irrespective of sleep. Thus, finding a Time $\times$ Emotion interaction for item memory after a phase of wakefulness would indicate that our effect originates from sleep-independent consolidation mechanisms, whereas a lack of a significant interaction would be a strong argument in favor of the sleep-specificity of our item memory effect. Secondly, with respect to our source memory results, it is of interest to test whether the differential pattern of memory benefits for neutral and emotional images we observed is uniquely tied to the respective night halves, specifically, the benefit of emotional source memory in the second night half. Notably, the decrease in emotional source performance in the early sleep group $\left(M_{\text {Diff }}=.06\right), F(1,16)=8.24, p=.011$, was considerably smaller than the deterioration of neutral source a

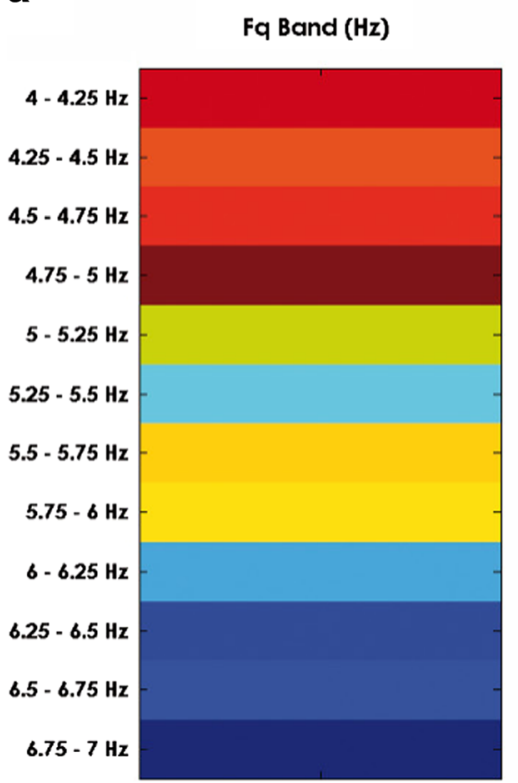

Correlational strength (Pearson's r)

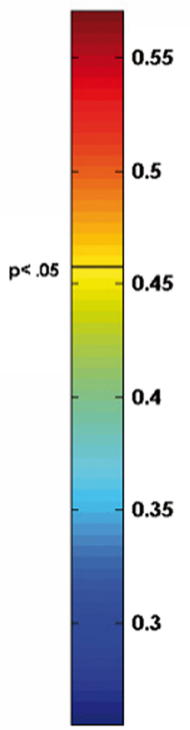

Fig. 6 (a) Correlations between emotional source memory (postsleep) in the late sleep group and right-frontal theta lateralization during REM sleep for each $0.25 \mathrm{~Hz}$ bin. Note. Correlation strength is represented by

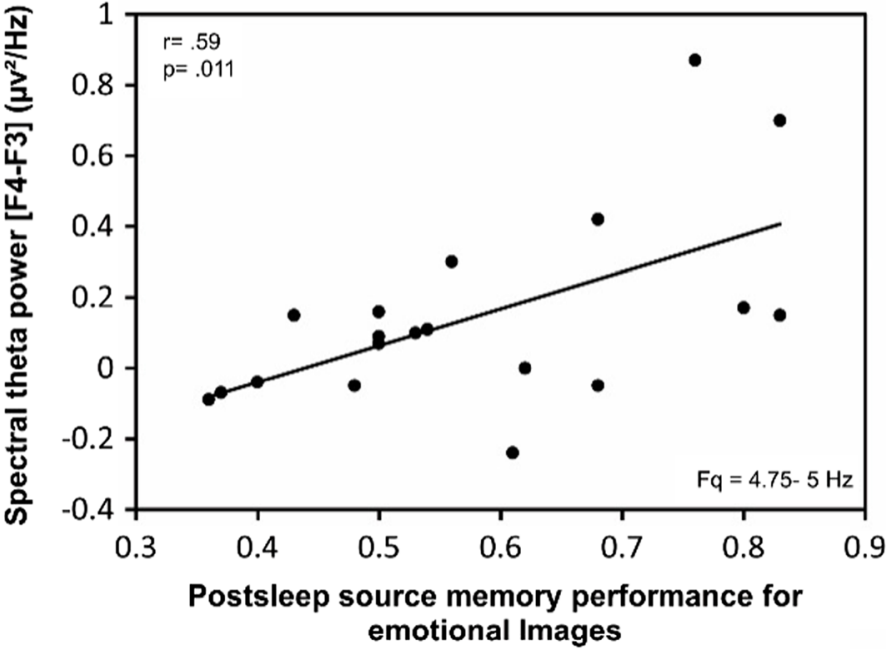

the color range. Warmer colors represent higher correlational strength. (b) Scatterplot of peak correlation in the $4.75-5 \mathrm{~Hz}$ bin. Zero-order correlation is depicted for greater comprehensibility (Color figure online) 
Table 4 Partial correlations between spindle power during SWS and postsleep memory performance

\begin{tabular}{|c|c|c|c|c|c|c|c|c|c|}
\hline \multirow[b]{3}{*}{ Sleep parameters } & & \multicolumn{4}{|c|}{ Early sleep group $(N=19)$} & \multicolumn{4}{|c|}{ Late sleep group $(N=19)$} \\
\hline & & \multicolumn{2}{|c|}{ Item memory } & \multicolumn{2}{|c|}{ Source memory } & \multicolumn{2}{|c|}{ Item memory } & \multicolumn{2}{|c|}{ Source memory } \\
\hline & & Emo & Neut & Emo & Neut & Emo & Neut & Emo & Neut \\
\hline \multirow[t]{7}{*}{ Slow spindle power $(11-13 \mathrm{~Hz})$} & F3 & $\begin{array}{l}.09 \\
p=.723\end{array}$ & $\begin{array}{l}.61^{*} \\
p=.007\end{array}$ & $\begin{array}{l}.07 \\
p=.777\end{array}$ & $\begin{array}{l}.22 \\
p=.386\end{array}$ & $\begin{array}{l}-.05 \\
p=.857\end{array}$ & $\begin{array}{l}.05 \\
p=.850\end{array}$ & $\begin{array}{l}-.24 \\
p=.348\end{array}$ & $\begin{array}{l}-.09 \\
p=.733\end{array}$ \\
\hline & $\mathrm{F} 4$ & $\begin{array}{l}.13 \\
p=.601\end{array}$ & $\begin{array}{l}.57 * \\
p=.013\end{array}$ & $\begin{array}{l}.01 \\
p=.976\end{array}$ & $\begin{array}{l}.18 \\
p=.480\end{array}$ & $\begin{array}{l}-.15 \\
p=.555\end{array}$ & $\begin{array}{l}-.04 \\
p=.865\end{array}$ & $\begin{array}{l}-.12 \\
p=.628\end{array}$ & $\begin{array}{l}-.04 \\
p=.864\end{array}$ \\
\hline & $\mathrm{C} 3$ & $\begin{array}{l}.03 \\
p=.905\end{array}$ & $\begin{array}{l}.67^{*} \\
p=.002\end{array}$ & $\begin{array}{l}.09 \\
p=.729\end{array}$ & $\begin{array}{l}.27 \\
p=.282\end{array}$ & $\begin{array}{l}-.13 \\
p=.604\end{array}$ & $\begin{array}{l}.01 \\
p=.975\end{array}$ & $\begin{array}{l}-.02 \\
p=.942\end{array}$ & $\begin{array}{l}.16 \\
p=.530\end{array}$ \\
\hline & $\mathrm{C} 4$ & -.04 & $.57 *$ & .18 & .33 & -.16 & .03 & -.06 & .07 \\
\hline & & $p=.866$ & $p=.014$ & $p=.489$ & $p=.184$ & $p=.515$ & $p=.895$ & $p=.819$ & $p=.782$ \\
\hline & $\mathrm{Cz}$ & .00 & $.58^{*}$ & .14 & .30 & -.42 & -.32 & .01 & .01 \\
\hline & & $p=.986$ & $p=.011$ & $p=.577$ & $p=.226$ & $p=.085$ & $p=.193$ & $p=.957$ & $p=.969$ \\
\hline \multirow[t]{6}{*}{ Fast spindle power $(13-15 \mathrm{~Hz})$} & F3 & .08 & $.58^{*}$ & .01 & .08 & .25 & .17 & -.27 & -.07 \\
\hline & & $p=.765$ & $p=.012$ & $p=.968$ & $p=.757$ & $p=.316$ & $p=.494$ & $p=.286$ & $p=.787$ \\
\hline & F4 & -.10 & .54 & .05 & .24 & .10 & .01 & -.09 & -.03 \\
\hline & & $p=.706$ & $p=.020$ & $p=.841$ & $p=.346$ & $p=.682$ & $p=.982$ & $p=.725$ & $p=.912$ \\
\hline & $\mathrm{C} 3$ & -.23 & .43 & .03 & .09 & .03 & .12 & -.05 & .17 \\
\hline & & $p=.353$ & $p=.073$ & $p=.923$ & $p=.736$ & $p=.902$ & $p=.651$ & $p=.842$ & $p=.493$ \\
\hline \multirow[t]{4}{*}{ Fast spindle power $(13-15 \mathrm{~Hz})$} & $\mathrm{C} 4$ & -.14 & .39 & .12 & .25 & .16 & .15 & -.16 & .10 \\
\hline & & $p=.586$ & $p=.115$ & $p=.628$ & $p=.315$ & $p=.531$ & $p=.567$ & $p=.519$ & $p=.689$ \\
\hline & $\mathrm{Cz}$ & -.46 & .10 & .11 & .13 & -.28 & -.44 & -.08 & .01 \\
\hline & & $p=.056$ & $p=.699$ & $p=.676$ & $p=.611$ & $p=.259$ & $p=.066$ & $p=.739$ & $p=.966$ \\
\hline
\end{tabular}

Note . Emo = emotional; Neut= neutral. Pearson correlation coefficents are reported with their respective uncorrected significance levels. Asterisks indicate significant correlations following Benjamini-Hochberg correction allowing for 5\% false positives. Statistical comparisons (according to Eid et al., 2010) between the strength of spindle correlations with emotional and neutral item memory performance in the early sleep group for slow spindle power (SS) and fast spindle power (FS): $\mathrm{SS}(\mathrm{F} 3) Z_{r}=2.33, p=.010 ; \mathrm{SS}(\mathrm{F} 4) Z_{r}=1.93, p=.027 ; \mathrm{SS}(\mathrm{C} 3) Z_{r}=2.91, p=.002 ; \mathrm{SS}(\mathrm{C} 4) Z_{r}=2.64, p=.004$; $\mathrm{SS}(\mathrm{Cz}) Z_{r}=2.51, p=.006 ; \mathrm{FS}(\mathrm{F} 3) Z_{r}=3.01, p=.001$

memory after late sleep $\left(M_{\text {Diff }}=.10\right), F(1,16)=14.67, p=$ .001. As such, the passage of time may have resulted in a globally enhanced emotional source retention, which could have biased the results toward finding differential benefits for neutral and emotional memories across both night halves. However, finding a general decrease of source memory (main effect of time and no Time $\times$ Emotion interaction) over an

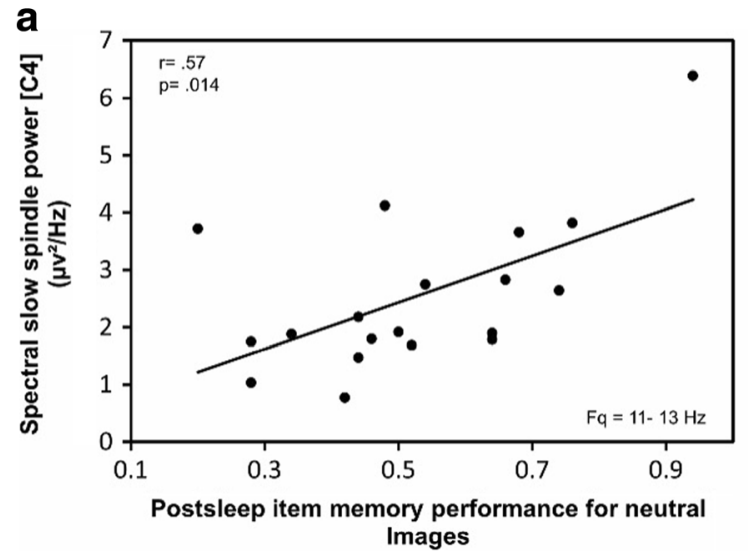

Fig. 7 Correlations between (a) slow spindle power $\left(\mu v^{2} / \mathrm{Hz}\right)$ at $\mathrm{C} 4$ and postsleep neutral item memory performance and (b) between fast spindle power $\left(\mu \mathrm{v}^{2} / \mathrm{Hz}\right)$ at $\mathrm{F} 3$ and retention benefit of neutral item memory relative to emotional item memory $\left(\right.$ PrNeut $_{\text {post }}-$ PrEmo $_{\text {post }}$ ) in the early identical interval of wakefulness would be strong support for our interpretation.

A comprehensive account of the design and statistical results of Experiment 2 can be found in the supplementary materials (see Table 5 for a summary of performance rates). In brief, emotional item memory was not modulated differentially across an equal interval of wakefulness, Time $\times$

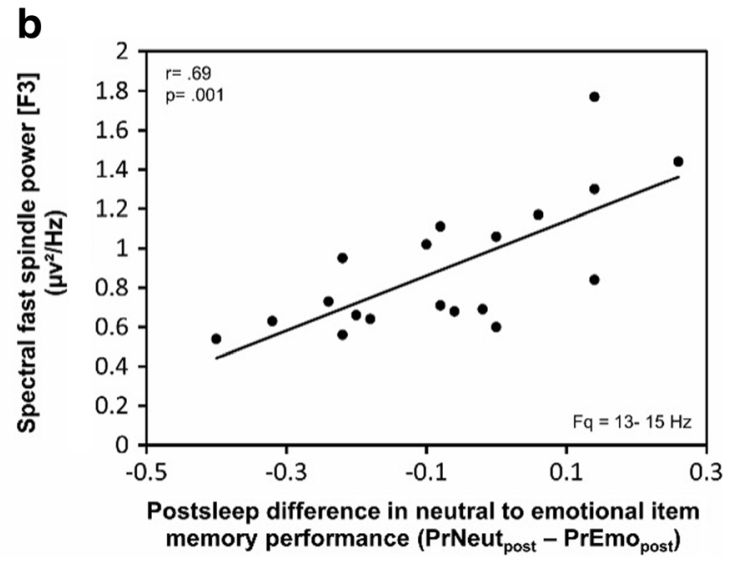

sleep group. Note. Zero-order correlations are depicted for greater comprehensibility. Benjamini-Hochberg correction was applied allowing for $5 \%$ false positives to the correlation depicted in (a) 
Table 5 Means of memory performance in Experiment 2 for item and source memory

\begin{tabular}{llll}
\hline Memory test & \multirow{2}{*}{ Performance measure } & \multicolumn{2}{l}{ Experiment $2(n=18)$} \\
\cline { 3 - 4 } & & Pretest & Posttest \\
\hline Item memory & $\mathrm{PR}_{\text {Emo }}$ & $.72(.03)$ & $.58(.03)$ \\
& $\mathrm{PR}_{\text {Neut }}$ & $.66(.03)$ & $.53(.05)$ \\
& Hits & $.85(.02)$ & $.77(.02)$ \\
& $\mathrm{Hits}_{\mathrm{Neut}}$ & $.76(.03)$ & $.64(.04)$ \\
& $\mathrm{FA}_{\text {Emo }}$ & $.14(.03)$ & $.19(.03)$ \\
& $\mathrm{FA}_{\text {Neut }}$ & $.10(.02)$ & $.11(.03)$ \\
Source memory & $\mathrm{SM}_{\text {Emo }}$ & $.59(.03)$ & $.49(.03)$ \\
& $\mathrm{SM}_{\text {Neut }}$ & $.60(.03)$ & $.51(.03)$ \\
\hline
\end{tabular}

Note. Hits = correctly recognized images of the item memory procedure; $\mathrm{FA}=$ false alarms to new images of the item memory procedure; $\mathrm{SM}=$ correctly recognized locations of items that had been correctly recognized in the preceding item memory procedure (correct source/overall hits). Standard errors of the mean are given in parentheses

Emotion: $F(1,17)=.001, p=.976$, Experiment 2, as across periods of sleep, Time $\times$ Emotion: $F(1,34)=9.24, p=.005$, main experiment. However, a main effect of emotion was similarly evident in Experiment 2, $F(1,17)=5.91, p=.026$, as in the former main experiment, $F(1,34)=13.88, p=.001$. Regarding source memory performance, a significant decline emerged across wakefulness, time: $F(1,17)=12.92, p=.002$, without any differential retention advantage for emotional or neutral stimuli, Time $\times$ Emotion: $F(1,17)=0.12, p=.736$. This marked decrease across both valence categories is in favor of a sleep-specific interpretation of the retention differences in source memory in our main study and does not suggest the involvement of circadian or time-dependent factors (for details, see Supplementary Data 3).

\section{Discussion}

On the basis of prior findings, we aimed to explore by which mechanisms sleep modulates the consolidation of emotional memories. Although REM sleep has been consistently found to exert a beneficial effect on emotional item memory, a possible involvement in the retention of emotion source memory has not been examined extensively to date. Consequently, we tested whether emotional source memory is supported by SWS, similar to source memory for neutral material, or whether emotional source memory is supported by REM sleepdependent consolidation mechanism, as has been demonstrated for emotional item memory retention. Performance decrements were contrasted across early and late night sleep for neutral and emotional images. Emotional images were both high in arousal and low in valence (negative), as confirmed by the results of a preceding pilot study.
In accordance with former accounts of a selective beneficial effect of sleep on emotional item memory, we found item memory for negative images to be preferentially retained across an interval of sleep as compared to neutral item memory. Surprisingly, however, this effect was not modulated by the early/late sleep manipulation. With respect to source memory performance, we found retention of negative and neutral material to be differentially modulated across early and late night sleep. In line with the well-characterized memory function of SWS (Diekelmann \& Born, 2010), source memory for neutral images was found to be selectively preserved across early night SWS-rich sleep. Late night REM sleep-rich sleep, on the other hand, was associated with a selective preservation of source memory for negative images. Moreover, the critical role of REM sleep in establishing this effect was confirmed by a significant correlation between emotional source memory at posttest and right-frontal theta lateralization in the late sleep group. On the other hand, spindle power during SWS exhibited selective correlations with item memory for neutral images in the early sleep group. These selective correlations are suggestive of a dissociation between emotional and neutral memory retention not just regarding early (SWS-rich) and late (REM sleep-rich) sleep, but also with respect to specific neurophysiological features of the respective sleep stages (theta oscillations during REM sleep and spindle power during SWS).

\section{Item memory}

Despite the absence of a clear dissociation with respect to early and late sleep, we did find emotional item memory to be selectively preserved across time, as opposed to item memory for neutral images. Moreover, the results of Experiment 2 demonstrated no significant interaction of time and emotion after three hours of wakeful retention. This lack of a retention benefit may suggest that the time-dependent advantage in retrieving emotional images was specifically related to sleep and does not reflect generic consolidation benefits over time (Bergado, Lucas, \& Richter-Levin, 2011; Sharot \& Yonelinas, 2008; Yonelinas \& Ritchey, 2015). Nonetheless, it must be acknowledged that a main effect of emotion on item memory was evident in Experiment 2, which suggests that certain aspects of preferential processing of emotional material were initiated irrespective of sleep or continued wakefulness in the critical consolidation phase. Consequently, the present results cannot fully disclose how and by which exact mechanism preferential retention of emotional item memory was established across time. Interestingly, the majority of former studies yielding time-dependent effects on emotional item memory entailed retention intervals of at least 24 hours, thus including a full night of sleep (Anderson, Yamaguchi, Grabski, \& Lacka, 2006; Nishida et al., 2009; Ritchey, Dolcos, \& Cabeza, 2008; Sharot \& Phelps, 2004; Sharot \& Yonelinas, 2008; Ventura-Bort et al., 2016; Wang, 2014; 
Weymar, Löw, \& Hamm, 2011; but see also LaBar \& Phelps, 1998; Rimmele, Davachi, Petrov, Dougal, \& Phelps, 2011). This pattern of former findings lends support to the notion of a time-dependent advantage that unfolds specifically as a result of sleep-related consolidation, which was similarly found in previous sleep studies (Groch et al., 2013; Groch et al., 2015; Nishida et al., 2009; Wagner et al., 2001). Although the exact mechanisms underlying the preferential retention of emotional item memory as well as the neural underpinnings require further exploration, the present results affirm the critical role of consolidation processes in establishing these effects.

The analyses of recollection-based and familiarity-based recognition in the present item memory procedure revealed a significant enhancement of $\mathrm{R}$ judgments for emotional stimuli, irrespective of time of testing. This is consistent with prior reports of an enhanced recollective experience for emotionally salient material (Johansson, Mecklinger, \& Treese, 2004; Ochsner, 2000; Rimmele et al., 2011) independent of sleeprelated consolidation mechanisms (Atienza \& Cantero, 2008; Sterpenich et al., 2007). In addition, as for item memory in general, $\mathrm{K}$ judgments to emotional items were selectively preserved over time as compared to $\mathrm{K}$ judgments to neutral items. This suggests that the time-dependent advantage that was revealed in the former item memory analysis (see above) is to be attributed to preserved familiarity-based recognition rather than recollection of emotional images. Conversely, this may point toward a consolidation benefit for emotional material that emerged independently of hippocampal memory processes. This interpretation would conform to frequent findings of time-dependent advantages in emotional item memory that do not generalize to performance in associative memory tests, which require recollective processing (see Sharot \& Yonelinas, 2008; Yonelinas \& Ritchey, 2015). On the other hand, with reference to the potential involvement of sleeprelated consolidation in establishing this effect, a study by $\mathrm{Hu}$ et al. (2006) revealed a highly similar pattern of results. Across a 12-hour sleep/wake design, the authors found a selective sleep-related enhancement of familiarity-based recognition of emotional images that was not evident in corresponding analyses of $\mathrm{R}$ judgments. However, as the critical mechanism of the time-dependent advantage in item memory and its neural underpinning requires further clarification, this is similarly the case with regard to the present findings of enhanced familiarity-based recognition of emotional material emerging across time.

\section{Source memory}

Examining the effects of early SWS-rich sleep and late REM sleep-rich sleep on source memory performance revealed a significant three-way interaction that suggests differential beneficial effects of early and late sleep for source memory of both valence categories. We found neutral source memory to be selectively preserved after early night SWS-rich sleep, which is generally in line with the active system consolidation account (Diekelmann \& Born, 2010). Moreover, we found a similar preservation of emotional source memory over late night REM sleep-rich sleep, which extends previous findings and points to a specific role of REM sleep in the consolidation of emotional source memory.

Our first finding raises the question of why early SWS-rich sleep did not result in a similar beneficial effect on emotional source memory (for similar results, see Alger \& Payne, 2016, and Groch et al., 2015), as would be expected if SWSdependent off-line processing provided a generalized function in associative memory consolidation (Diekelmann \& Born, 2010). Based on the finding that sleep-dependent benefits on associative memory only emerge when participants receive instructions about the subsequent test phase prior to encoding (Wilhelm et al., 2011), one may assume that the learning intention during encoding acts as the critical "tagging" mechanism, which determines whether sleep-related system consolidation is deployed. Such a specific learning experience, characterized by intentional encoding and the engagement of control processes, may be associated with activation in the prefrontal cortex (Ranganath, 2010), which, in turn, contributes to the generation of slow oscillations and thus potentially impacts hippocampal reactivations during SWS (Batterink, Creery, \& Paller, 2016; Diekelmann \& Born, 2010). The salience of emotional stimuli may attenuate these tagging mechanisms during encoding as the material is perceived as highly distinctive and easily memorable, seemingly requiring less effort to successfully retain the item and its source feature (see Hourihan, Fraundorf, \& Benjamin, 2017, for evidence that emotional material influences metamnemonic judgments in this manner). On the other hand, neutral material may be perceived as less conspicuous, thus engaging more strategic processing mechanisms during intentional encoding. In line with this framework, selective benefits of sleep on neutral item memory performance have been found to rely on explicit encoding conditions, whereas these do not unfold after incidental learning (Bennion et al., 2016; but see Cunningham, Chambers, \& Payne, 2014a). Moreover, the assumption that selective benefits for neutral source memory originate from SWS-dependent memory processing has been originally substantiated by Groch et al. (2015). The authors reported an enhancing effect of early night SWS-rich sleep on neutral source memory retention but not on emotional source memory retention in a similar split-night study design. However, when the motivational value of the material was additionally varied (Study 2), a general retention benefit of both emotional and neutral source memory over early night sleep emerged. Thus, the presumed enhancement of learning intention by motivational cues may have led to a similar and strong recruitment of tagging mechanisms during the encoding of emotional stimuli, as in the case of neutral stimuli. Consequently, this may 
have resulted in an equal initiation of system consolidation processes during SWS for neutral and emotional material. Notably, a recent study by Lehmann, Schreiner, Seifritz, and Rasch (2016) revealed a selective cueing benefit for emotional image-word associations across NREM sleep, which can be integrated with the aforementioned view. In this study, participants encoded associations between emotional or neutral images and auditorily presented words. Thereafter, participants were exposed to half of the previously encoded auditory cues either during NREM sleep in the first night half or during REM sleep in the second night half. In the NREM sleep cueing condition, the authors found that uncued neutral associations exhibited similar levels of memory retention as those that were cued during sleep. This pattern of results may reflect that neutral associations successfully engaged tagging mechanisms during encoding and were thus spontaneously reactivated during SWS without requiring external enforcement by means of auditory cueing. Consequently, this emergence of spontaneous reactivations in the uncued condition may have resulted in an absence of retention differences between uncued and cued neutral associations. Emotional associations, on the other hand, were retained to a significantly higher extent after auditory cueing. Thus, it may be inferred that emotional images attenuated tagging mechanisms at the encoding stage resulting in a strong benefit of respective associations from external cueing during NREM sleep. In other words, when emotional associations are enforced by motivational cues (as in Study 2 of Groch et al., 2015) or directly cued during NREM sleep (as in Lehmann et al., 2016), they gain access to SWS-related processing, whereas without such an external enforcement (as in the present study, in Study 1 of Groch et al., 2015, and in the uncued item category of Lehmann et al., 2016), emotional associations do not exhibit preferential retention across early night SWS-rich sleep.

Although we did not find a beneficial effect of SWS on emotional source memory retention in the present study, possibly due to the factors outlined above, we did nevertheless find a selective preservation of emotional source memory over late REM sleep-rich sleep. This is a novel finding and suggests that the well-characterized retention benefit in emotional item memory observed in association with REM sleep (but see Baran et al., 2012; Cairney et al., 2013; Cairney et al., 2015) extended to the retention of source features of emotional material under the present testing conditions. In conjunction with the highly specific correlation between REM sleep's neurophysiological features and postsleep emotional source memory performance (see below for further discussion), we believe that the underlying process of consolidation may operate in a manner that is highly distinctive and distinguishable from SWS-related system consolidation. The prerequisite to this process may be the successful perceptually based integration of the respective item and its associative features at the encoding stage (Chiu et al., 2013; Mather, 2007), which is assumed to be facilitated by emotion under certain conditions (Kensinger, 2009). Recent accounts in the literature suggest that emotional associations are preferentially retained only when they entail features that are "intrinsic", thus, perceptually inherent to the stimulus (such as color or location), whereas the process of associative binding appears to be impaired for "extrinsic" features (Chiu et al., 2013; Kensinger, 2009; Mather, 2007). Taking into account that REM sleep-related emotional memory processing may only occur as a sequel of arousal-induced changes in synaptic plasticity during initial encoding (Bergado et al., 2011; Richter-Levin \& Akirav, 2003), enhancing effects of REM sleep may thus be limited to intrinsic source features that previously underwent emotion-facilitated perceptual integration. In support of this notion, recent findings by Bennion, Payne, and Kensinger (2017) establish a relationship between activation changes along the ventral visual stream during successful retrieval of emotionally associated material and preceding REM sleep duration. Although requiring further investigation, these effects may indeed reflect a unique association between REM-sleepdependent consolidation and emotion-driven modulations in perceptual processing.

The assumption that consolidation driven by emotional salience is limited to source features in spatiotemporal proximity of the emotional item could plausibly account for the absence of REM sleep-related effects in previous studies (Groch et al., 2015; Lehmann et al, 2016). In the study of Lehman et al. (2016), cueing emotional associations during REM sleep did not elicit any significant retention benefit as contrasted with uncued associations. However, as the study employed associations between visually presented images and auditorily presented words, this inherently requires associative binding across different modalities (Mayes, Montaldi, \& Migo, 2007), which may not be accomplished by the perceptual binding mechanism proposed above. In a similar vein, specific aspects of the task design employed by Groch et al. (2015) may have prevented facilitating effects of late night sleep on emotional source memory from emerging. The task design varied two independent features (location and color) simultaneously and presented one of these features (color) under spatiotemporally discontiguous conditions. This procedure likely resulted in enhanced attentional demands during encoding and may thus have been less suited to ascertain potential effects of emotion on perceptual integration. The current task design, on the other hand, deviates importantly from Groch et al. (2015) as it employed a single feature (location) as well as interactive encoding instructions. Processes of emotion-facilitated perceptual integration may thus have emerged with greater clarity in the current task procedure, which may account for different results in the late sleep condition of both experiments.

From a broader perspective, the coexistence of two consolidation processes, one driven by learning intention and the other driven by emotional salience, would be highly adaptive, 
as integral features of emotional events would be consolidated in any instance, while neutral associations would require the engagement of learning intention to be successfully retained across time. Although this line of interpretation requires further elaboration in future studies, the concept of independent processing mechanisms related to REM sleep and SWS receives additional support from the current neurophysiological findings. Thus, we were able to further substantiate behavioral differences in emotional source memory performance by means of a partial neurophysiological dissociation regarding specific oscillatory characteristics of REM sleep.

\section{Neurophysiological correlates of memory consolidation}

Source memory retrieval of emotional images after sleeping was significantly and positively correlated with right-frontal theta lateralization in the late sleep group. This association strongly substantiates that specific processes during REM sleep are critical for emotional memory consolidation rather than general effects of time or sleep. The laterality of this effect at frontal recording sites merges with findings of episodic memory encoding and retrieval being related to activation of the right dorsolateral prefrontal cortex (Rugg, Otten, \& Henson, 2002) especially for complex pictorial stimuli (Brewer, Zhao, Desmond, Glover, \& Gabrieli, 1998). Emotional processing of pictorial or image-related material has conversely been linked to right-lateralized amygdala activation (McMenamin \& Marsolek, 2013). Furthermore, hemispheric asymmetry in frontal theta activity has generally been found to be a feature of REM sleep (Bolduc, Daoust, Limoges, Braun, \& Godbout, 2003; Vyazovskiy, Borbély, \& Tobler, 2002). In the present study, subsequent analysis in each frequency bin revealed peak correlations in the lower theta frequency range $(4-5 \mathrm{~Hz})$. This distribution of correlations corroborates the proposition of a lower frequency "slow" theta activity occurring in the human hippocampus during both wake and REM sleep (Hutchison \& Rathore, 2015; Jacobs, 2014; Lega, Jacobs, \& Kahana, 2012). This slower theta activity $(\sim 3 \mathrm{~Hz})$ is considered to be more physiologically analogous to faster theta in rodents $(4-10 \mathrm{~Hz})$, which is often implied to reflect encoding-related activity during the acquisition of new episodes (Hutchison \& Rathore, 2015). Taken together, the current findings, although preliminary, are in line with the notion that REM sleep may provide a critical window for consolidation processes of emotionally salient episodes in the medial temporal lobe memory system and thus support associative memory retention for this specific category of events (Boyce et al., 2016; Hutchison \& Rathore, 2015). However, future studies are required to disentangle the detailed mechanisms by which prefrontal theta oscillations may mediate the consolidation of emotional source memory and to elaborate on the functional significance of hemispherical asymmetries in this context.
With regard to the neurophysiological correlates of SWSdependent consolidation, we found significant correlations between frontocentral slow spindle power as well as frontal (F3) fast spindle power and postsleep memory performance. These results are in line with prior studies showing that sleep spindle density constitutes a sensitive measure of system consolidation processes occurring during NREM sleep (Mednick et al., 2013; Studte et al., 2015; Studte et al., 2017). It is assumed that temporally coupled spindle-ripple events provide a physiological mechanism for a fine-tuned hippocampal-neocortical information flow (Diekelmann \& Born, 2010). Remarkably, we found selective correlations of spindle power with item memory performance for neutral images, but not for emotional images, affirming our behavioral results of a selective enhancement of neutral memory retention over early night SWS-rich sleep. However, contrary to our hypothesis, this association was only evident for item memory performance and not for neutral source memory. This correlation does not parallel behavioral findings but, nonetheless, strengthens the notion of SWS selectively benefitting neutral memory representations. Also, it must be taken into account that item and source memory were assessed in a combined procedure within the same test phase. Although we aimed to control for shared variance of item and source memory, our procedure may have created an overlap in both measures resulting in stronger correlations between item and source memory performance than in paradigms in which memory for items and associations are independently manipulated (e.g. Studte et al., 2015).

In summary, our results demonstrate a broadly consistent dissociation between neutral and emotional memory retention with respect to consolidation processes of both night halves, which was evident in both behavioral and neurophysiological measures. Admittedly, differential retention benefits in our behavioral analyses were of modest size and thus did not result in any postsleep differences in source memory performance between groups. However, it is important to consider that the restricted sample size in combination with the between-subject manipulation likely resulted in high levels of subject-specific variance. We specifically aimed to reduce the impact of this variance in our subsequent analysis by introducing covariate measures of general (neuro-)cognitive abilities. Nonetheless, other unaccounted sources of variance between subjects may have precluded finding significant differences in direct between-group comparisons. Thus, the present effects should be regarded as preliminary and require further substantiation in future experiments. Future studies employing the split-night-procedure should particularly seek to include a wake control group in order to exhaustively account for potential effects related to the passage of time. Although the lack of differential decrements in memory performance in Experiment 2 substantiates our interpretation with regard to the findings of our main experiment, these require replication in a combined sleep-wake design. Contrasting performance decrements across wakefulness to sleep-related consolidation may also yield more pronounced differences between 
groups, which were not attainable in the nuanced comparison of SWS-rich and REM sleep-rich sleep across both night halves.

\section{Emotional reactivity to previously encoded images}

Although we found REM sleep to be beneficial for memory retention of emotionally salient information, we found an opposing effect on emotional reactivity. Participants of the late sleep group, as compared to the early sleep group, rated emotional images to be more arousing irrespective of whether these had been studied before sleep (hits) or encountered as lures in the postsleep retrieval test (correct rejections), thus first presented after sleep. Our results consequently contradict former conceptions of REM sleep-related off-line memory processing (Goldstein \& Walker, 2014; Walker \& van der Helm, 2009), which postulate that REM sleep-dependent consolidation processes are not just beneficial to emotional memory retention but also result in a concomitant reduction of reactivity toward reprocessed stimuli. Conversely, the effect which emerged in the current analysis may indicate that reactivity toward emotional stimuli is generally enhanced, rather than attenuated, subsequent to REM sleep-related processing, which is in line with a growing body of literature demonstrating similar effects (Baran et al., 2012; Werner et al., 2015). We do not assume that the circadian discrepancy between early and late sleep groups can account for these unpredicted findings. General circadian influences, if present, should be apparent with regard to ratings of both emotional and neutral stimuli and on both stimulus dimensions (valence and arousal), which was not the case. Future studies focusing on a more comprehensive assessment of emotional reactivity by means of objective and subjective parameters are thus required to further elucidate by which mechanism and in which particular direction emotional reactivity is modulated across periods of REM sleep.

\section{Methodological considerations}

Even though split-night designs are an established procedure to compare consolidation effects related to SWS and REM sleep, respectively, they also bear some inherent limitations. First, circadian influences arising from the split-night manipulation are to be considered in the interpretation of findings on memory performance. Indeed circadian influences and concomitant rises in cortisol levels have been shown to modulate emotional learning (Lass-Hennemann \& Michael, 2014; van Ast, Cornelisse, Meeter, Joëls, \& Kindt, 2013), albeit with an inhibiting effect during memory retrieval (Bentz et al., 2013; Diekelmann, Wilhelm, Wagner, \& Born, 2011). Consequently, the rise of cortisol levels across the second night half should have resulted in a detrimental effect on emotional memory performance rather than the observed relative retention benefit for emotional source memory. Secondly, it may be argued that the night half of sleep preceding encoding in the late group may impact comparability between both groups regarding processes of initial encoding. In fact, SWS appears to subserve synaptic homeostasis and hence subsequent learning by providing a window for synaptic downscaling (Tononi \& Cirelli, 2003). However, these influences should have been evident immediately following encoding, which was not the case, as groups were closely comparable in performance measures at pretest (see a priori group comparisons). Despite these limitations, the split-night design offers a unique approach by enabling to contrast two sleep conditions that merely differ in the relative amount of time spent in SWS and REM sleep, respectively. Hence, resulting behavioral differences are not prone to involve effects of interference or generic neurophysiological regeneration, as they likely occur in studies contrasting the effects of sleep against wakefulness (e.g., nap designs). As such, we believe that the present study adds significantly to the existing literature by providing preliminary evidence of a sleep-dependent effect on emotional source memory in association with a highly specific neurophysiological feature of REM sleep (right-frontal theta lateralization). Importantly, this evidence was established in a design that contrasts memory retention processes under conditions that are closely comparable in the critical consolidation phase.

From a broader perspective, it is interesting to point out that neurophysiological characteristics of REM sleep (e.g., theta power during REM sleep) have been found to differ in individuals suffering from posttraumatic stress disorder. These observations open up the possibility that highly specific mechanisms in emotional memory consolidation, which emerged in the current findings, may be of relevance in the development of psychopathological conditions (Cowdin, Kobayashi, \& Mellman, 2014). Beyond this, our results affirm the critical relevance of initial conditions and processes during encoding in determining subsequent reprocessing, the involvement of different structures and, lastly, the outcomes of sleep-related memory consolidation. The complex interactions resulting from this are only beginning to be understood and open up intriguing perspectives for future study.

Acknowledgements We gratefully acknowledge Leonie Lonsdorfer, Raphaela Müller, Verena Pistner, Kevin Rozario, Ananda Möllenhof, Wencke Lubojanski, and Lorraine Rosenberger for assisting with data collection/analysis. Further, we would like to thank Marlene Staginnus for proofreading the manuscript.

Funding This research was supported by a grant from the German Research Foundation (IRTG 1457).

\section{References}

Ackermann, S., Hartmann, F., Papassotiropoulos, A., de Quervain, D., \& Rasch, B. (2015). No associations between interindividual differences in sleep parameters and episodic memory consolidation. Sleep, 38(6), 951-959.

Alger, S. E., \& Payne, J. D. (2016). The differential effects of emotional salience on direct associative and relational memory during a nap. Cognitive, Affective, \& Behavioral Neuroscience, 16(6), 1150-1163. 
American Academy of Sleep Medicine. (2007). AASM manual for the scoring of sleep and associated events: Rules, terminology and technical specifications. Darien, IL: Author.

Anderson, A. K., Yamaguchi, Y., Grabski, W., \& Lacka, D. (2006). Emotional memories are not all created equal: Evidence for selective memory enhancement. Learning \& Memory, 13(6), 711-718.

Antony, J. W., Ferreira, C. S., Norman, K. A., \& Wimber, M. (2017). Retrieval as a fast route to memory consolidation. Trends in Cognitive Sciences. 21(8), 573-576.

Atienza, M., \& Cantero, J. L. (2008). Modulatory effects of emotion and sleep on recollection and familiarity. Journal of Sleep Research, 17(3), 285-294.

Baran, B., Daniels, D., \& Spencer, R. M. (2013). Sleep-dependent consolidation of value-based learning. PLOS ONE, 8(10), e75326.

Baran, B., Pace-Schott, E. F., Ericson, C., \& Spencer, R. M. (2012). Processing of emotional reactivity and emotional memory over sleep. Journal of Neuroscience, 32(3), 1035-1042.

Barner, C., Seibold, M., Born, J., \& Diekelmann, S. (2017). Consolidation of prospective memory: Effects of sleep on completed and reinstated intentions. Frontiers in Psychology, 7, 2025.

Batterink, L. J., Creery, J. D., \& Paller, K. A. (2016). Phase of spontaneous slow oscillations during sleep influences memory-related processing of auditory cues. Journal of Neuroscience, 36(4), 1401-1409.

Benjamini, Y., \& Hochberg, Y. (1995). Controlling the false discovery rate: A practical and powerful approach to multiple testing. Journal of the Royal Statistical Society: Series B (Statistical Methodology), 57(1), 289-300.

Bennion, K. A., Payne, J. D., \& Kensinger, E. A. (2016). The impact of napping on memory for future-relevant stimuli: Prioritization among multiple salience cues. Behavioral Neuroscience, 130(3), 281-289.

Bennion, K. A., Payne, J. D., \& Kensinger, E. A. (2017). Residual effects of emotion are reflected in enhanced visual activity after sleep. Cognitive, Affective, \& Behavioral Neuroscience, 17(2), 290-304.

Bentz, D., Michael, T., Wilhelm, F. H., Hartmann, F. R., Kunz, S., von Rohr, I. R. R., \& de Quervain, D. J. F. (2013). Influence of stress on fear memory processes in an aversive differential conditioning paradigm in humans. Psychoneuroendocrinology, 38(7), 1186-1197.

Bergado, J. A., Lucas, M., \& Richter-Levin, G. (2011). Emotional tagging-a simple hypothesis in a complex reality. Progress in Neurobiology, 94(1), 64-76.

Bergstrom, Z. M., Henson, R. N., Taylor, J. R., \& Simons, J. S. (2013). Multimodal imaging reveals the spatiotemporal dynamics of recollection. Neurolmage, 68, 141-153.

Bolduc, C., Daoust, A.-M., Limoges, É., Braun, C. M. J., \& Godbout, R. (2003). Hemispheric lateralization of the EEG during wakefulness and REM sleep in young healthy adults. Brain and Cognition, 53(2), 193-196.

Boyce, R., Glasgow, S. D., Williams, S., \& Adamantidis, A. (2016). Causal evidence for the role of REM sleep theta rhythm in contextual memory consolidation. Science, 352(6287), 812-816.

Bradley, M. M., \& Lang, P. J. (1994). Measuring emotion: The selfassessment manikin and the semantic differential. Journal of Behavior Therapy and Experimental Psychiatry, 25(1), 49-59.

Brewer, J. B., Zhao, Z., Desmond, J. E., Glover, G. H., \& Gabrieli, J. D. E. (1998). Making memories: Brain activity that predicts how well visual experience will be remembered. Science, 281(5380), 1185-1187.

Buysse, D. J., Reynolds, C. F., Monk, T. H., \& Hoch, C. C. (1991). Quantification of subjective sleep quality in healthy elderly men and women using the Pittsburgh Sleep Quality Index (PSQI). Sleep, 14(4), 331-338.

Buzsáki, G. (2015). Hippocampal sharp wave-ripple: A cognitive biomarker for episodic memory and planning. Hippocampus, 25(10), $1073-1188$

Cairney, S. A., Durrant, S. J., Hulleman, J., \& Lewis, P. A. (2013). Targeted memory reactivation during slow wave sleep facilitates emotional memory consolidation. Sleep, 37(4), 701-707.
Cairney, S. A., Durrant, S. J., Jackson, R., \& Lewis, P. A. (2014). Sleep spindles provide indirect support to the consolidation of emotional encoding contexts. Neuropsychologia, 63, 285-292.

Cairney, S. A., Durrant, S. J., Power, R., \& Lewis, P. A. (2015). Complementary roles of slow-wave sleep and rapid eye movement sleep in emotional memory consolidation. Cerebral Cortex, 25(6), $1565-1575$.

Cellini, N., Torre, J., Stegagno, L., \& Sarlo, M. (2016). Sleep before and after learning promotes the consolidation of both neutral and emotional information regardless of REM presence. Neurobiology of Learning and Memory, 133, 136-144.

Chiu, Y.-C., Dolcos, F., Gonsalves, B. D., \& Cohen, N. J. (2013). On opposing effects of emotion on contextual or relational memory. Frontiers in Psychology, 4, 103.

Cousineau, D. (2005) Confidence intervals in within-subject designs: A simpler solution to Loftus and Masson's method. Tutorials in Quantitative Methods for Psychology, 1(1), 42-45.

Cowdin, N., Kobayashi, I., \& Mellman, T. A. (2014). Theta frequency activity during rapid eye movement (REM) sleep is greater in people with resilience versus PTSD. Experimental Brain Research, 232(5), $1479-1485$.

Cunningham, T. J., Chambers, A. M., Payne, J. D. (2014a) Prospection and emotional memory: how expectation affects emotional memory formation following sleep and wake. Frontiers in Psychology, 5, 862.

Cunningham, T. J., Crowell, C. R., Alger, S. E., Kensinger, E. A., Villano, M. A., Mattingly, S. M., \& Payne, J. D. (2014b). Psychophysiological arousal at encoding leads to reduced reactivity but enhanced emotional memory following sleep. Neurobiology of Learning and Memory, 114, 155-164.

Datta, S., O’Malley, M. W. (2013) Fear Extinction Memory Consolidation Requires Potentiation of Pontine-Wave Activity during REM Sleep. Journal of Neuroscience, 33(10), 4561-4569.

Daurat, A., Terrier, P., Foret, J., \& Tiberge, M. (2007). Slow wave sleep and recollection in recognition memory. Consciousness and Cognition, 16(2), 445-455.

Diekelmann, S., \& Born, J. (2010). The memory function of sleep. Nature Reviews Neuroscience, 11(2), 114-126.

Diekelmann, S., Wilhelm, I., Wagner, U., \& Born, J. (2011). Elevated cortisol at retrieval suppresses false memories in parallel with correct memories. Journal of Cognitive Neuroscience, 23(4), 772-781.

Diekelmann, S., Wilhelm, I., Wagner, U., \& Born, J. (2013). Sleep improves prospective remembering by facilitating spontaneousassociative retrieval processes. PLOS ONE, 8(10), 1-10.

Diener, E. (2000). Subjective well-being: The science of happiness and a proposal for a national index. American Psychologist, 55(1), 34-43.

Drosopoulos, S., Wagner, U., \& Born, J. (2005). Sleep enhances explicit recollection in recognition memory. Learning \& Memory, 12(1), 44-51.

Easterbrook, J. A. (1959). The effect of emotion on cue utilization and the organization of behavior. Psychological Review, 66(3), 183-201.

Eid, M., Gollwitzer, M., \& Schmitt, M. (2010). Statistik und Forschungsmethoden [Statistics and research methods]. Weinheim, Germany: Beltz.

Ekstrand, B. R. (1967). Effect of sleep on memory. Journal of Experimental Psychology, 75(1), 64-72.

Fischer, S., \& Born, J. (2009). Anticipated reward enhances offline learning during sleep. Journal of Experimental Psychology: Learning, Memory, and Cognition, 35(6), 1586-1593.

Formann, A. K., Waldherr, K., \& Piswanger, K. (2011). Wiener Matrizen Test-2 (WMT-2): Ein Rasch-skalierter sprachfreier Kurztest zur Erfassung der Intelligenz [Viennese Matrices Test: A Rasch-scaled culture-fair intelligence test]. Weinheim, Germany: Beltz.

Genzel, L., Kiefer, T., Renner, L., Wehrle, R., Kluge, M., Grözinger, M., ... Dresler, M. (2012). Sex and modulatory menstrual cycle effects on sleep related memory consolidation. Psychoneuroendocrinology, 37(7), 987-998. 
Goldstein, A. N., \& Walker, M. P. (2014). The role of sleep in emotional brain function. Annual Review of Clinical Psychology, 10, 679-708.

Groch, S., Wilhelm, I., Diekelmann, S., \& Born, J. (2013). The role of REM sleep in the processing of emotional memories: Evidence from behavior and event-related potentials. Neurobiology of Learning and Memory, 99, 1-9.

Groch, S., Zinke, K., Wilhelm, I., \& Born, J. (2015). Dissociating the contributions of slow-wave sleep and rapid eye movement sleep to emotional item and source memory. Neurobiology of Learning and Memory, 122, 122-130.

Heib, D. P., Hoedlmoser, K., Anderer, P., Gruber, G., Zeitlhofer, J., \& Schabus, M. (2015). Oscillatory theta activity during memory formation and its impact on overnight consolidation: A missing link? Journal of Cognitive Neuroscience, 27(8), 1648-1658.

Hoddes, E., Zarcone, V., Smythe, H., Phillips, R., \& Dement, W. C. (1973). Quantification of sleepiness: A new approach. Psychophysiology, 10(4), 431-436.

Hourihan, K. L., Fraundorf, S. H., \& Benjamin, A. S. (2017). The influences of valence and arousal on judgments of learning and on recall. Memory \& Cognition, 45(1), 121-136.

Hu, P., Stylos-Allan, M., \& Walker, M. P. (2006). Sleep facilitates consolidation of emotional declarative memory. Psychological Science, 17(10), 891-898.

Hutchison, I. C., \& Rathore, S. (2015). The role of REM sleep theta activity in emotional memory. Frontiers in Psychology, 6, 1439.

Iglói, K., Gaggioni, G., Sterpenich, V., \& Schwartz, S. (2015). A nap to recap or how reward regulates hippocampal-prefrontal memory networks during daytime sleep in humans. eLife, 4, e07903.

Jacobs, J. (2014). Hippocampal theta oscillations are slower in humans than in rodents: Implications for models of spatial navigation and memory. Philosophical Transactions of the Royal Society B, 369(1635). https://doi.org/10.1098/rstb.20130304

Johansson, M., Mecklinger, A., \& Treese, A.-C. (2004). Recognition memory for emotional and neutral faces: An event-related potential study. Journal of Cognitive Neuroscience, 16(10), 1840-1853.

Kensinger, E. A. (2009). Remembering the details: Effects of emotion. Emotion Review, 1(2), 99-113.

Kensinger, E. A., \& Corkin, S. (2004). Two routes to emotional memory: Distinct neural processes for valence and arousal. Proceedings of the National Academy of Sciences of the United States of America, 101(9), 3310-3315.

Kroenke, K., Spitzer, R. L., \& Williams, J. B. (2001). The PHQ-9. Journal of General Internal Medicine, 16(9), 606-613.

LaBar, K. S., \& Phelps, E. A. (1998). Arousal-mediated memory consolidation: Role of the medial temporal lobe in humans. Psychological Science, 9(6), 490-493.

Lang, P. J., Bradley, M. M., \& Cuthbert, B. N. (2005). International affective picture system (IAPS): Affective ratings of pictures and instruction manual. Gainesville, Florida: NIMH, Center for the Study of Emotion \& Attention.

Lass-Hennemann, J., \& Michael, T. (2014). Endogenous cortisol levels influence exposure therapy in spider phobia. Behaviour Research and Therapy, 60, 39-45.

Lega, B. C., Jacobs, J., \& Kahana, M. (2012). Human hippocampal theta oscillations and the formation of episodic memories. Hippocampus, 22(4), 748-761.

Lehmann, M., Schreiner, T., Seifritz, E., Rasch, B. (2016) Emotional arousal modulates oscillatory correlates of targeted memory reactivation during NREM, but not REM sleep. Scientific Reports, 6(1), 39229.

Lewis, P., Cairney, S., Manning, L., \& Critchley, H. (2011). The impact of overnight consolidation upon memory for emotional and neutral encoding contexts. Neuropsychologia, 49(9), 2619-2629.

Lo, J. C., Bennion, K. A., \& Chee, M. W. L. (2016). Sleep restriction can attenuate prioritization benefits on declarative memory consolidation. Journal of Sleep Research, 25(6), 664-672.
Marchewka, A., Żurawski, Ł., Jednoróg, K., \& Grabowska, A. (2014). The Nencki Affective Picture System (NAPS): Introduction to a novel, standardized, wide-range, high-quality, realistic picture database. Behavior Research Methods, 46(2), 596-610.

Mather, M. (2007). Emotional arousal and memory binding: An object-based framework. Perspectives on Psychological Science, 2(1), 33-52.

Mayes, A., Montaldi, D., \& Migo, E. (2007). Associative memory and the medial temporal lobes. Trends in Cognitive Sciences, 11(3), 126-135.

McMenamin, B. W., \& Marsolek, C. J. (2013). Can theories of visual representation help to explain asymmetries in amygdala function? Cognitive, Affective, \& Behavioral Neuroscience, 13(2), 211-224.

Mednick, S. C., McDevitt, E. A., Walsh, J. K., Wamsley, E., Paulus, M., Kanady, J. C., \& Drummond, S. P. (2013). The critical role of sleep spindles in hippocampal-dependent memory: A pharmacology study. Journal of Neuroscience, 33(10), 4494-4504.

Menz, M. M., Rihm, J. S., \& Buchel, C. (2016). REM sleep is causal to successful consolidation of dangerous and safety stimuli and reduces return of fear after extinction. Journal of Neuroscience, 36(7), 2148-2160.

Miller, G. A., \& Chapman, J. P. (2001). Misunderstanding analysis of covariance. Journal of Abnormal Psychology, 110(1), 40-48.

Murray, B. D., \& Kensinger, E. A. (2013). A review of the neural and behavioral consequences for unitizing emotional and neutral information. Frontiers in Behavioral Neuroscience, 7, 1-9.

Nishida, M., Pearsall, J., Buckner, R. L., \& Walker, M. P. (2009). REM sleep, prefrontal theta, and the consolidation of human emotional memory. Cerebral Cortex, 19(5), 1158-1166.

Nuwer, M. R., Comi, G., Emerson, R., Fuglsang-Frederiksen, A., Guerit, J. M., Hinrichs, H., ... Rappelsburger, P. (1998). IFCN standards for digital recording of clinical EEG. International Federation of Clinical Neurophysiology. Electroencephalography and Clinical Neurophysiology, 106(3), 259-261.

O'Brien, F., \& Cousineau, D. (2014). Representing error bars in withinsubject designs in typical software packages. The Quantitative Methods for Psychology, 10, 58-70.

Ochsner, K. N. (2000). Are affective events richly recollected or simply familiar? The experience and process of recognizing feelings past. Journal of Experimental Psychology: General, 129(2), 242-261.

Oldfield, R. C. (1971). The assessment and analysis of handedness: The Edinburgh inventory. Neuropsychologia, 9(1), 97-113.

Park, D. C., Smith, A. D., Lautenschlager, G., Earles, J. L., Frieske, D., Zwahr, M., \& Gaines, C. L. (1996). Mediators of long-term memory performance across the life span. Psychology and Aging, 11(4), 621-637.

Payne, J. D., Chambers, A. M., \& Kensinger, E. A. (2012). Sleep promotes lasting changes in selective memory for emotional scenes. Frontiers in Integrative Neuroscience, 6, 1-11.

Payne, J. D., \& Kensinger, E. A. (2011). Sleep leads to changes in the emotional memory trace: evidence from FMRI. Journal of Cognitive Neuroscience, 23(6), 1285-1297.

Payne, J. D., Stickgold, R., Swanberg, K., \& Kensinger, E. A. (2008). Sleep preferentially enhances memory for emotional components of scenes. Psychological Science, 19(8), 781-788.

Plihal, W., \& Born, J. (1997). Effects of early and late nocturnal sleep on declarative and procedural memory. Journal of Cognitive Neuroscience, 9(4), 534-547.

Popa, D., Duvarci, S., Popescu, A. T., Léna, C., \& Paré, D. (2010). Coherent amygdalocortical theta promotes fear memory consolidation during paradoxical sleep. Proceedings of the National Academy of Sciences of the United States of America, 107(14), 6516-6519.

Prehn-Kristensen, A., Göder, R., Chirobeja, S., Breßmann, I., Ferstl, R., \& Baving, L. (2009). Sleep in children enhances preferentially emotional declarative but not procedural memories. Journal of Experimental Child Psychology, 104(1), 132-139.

Prehn-Kristensen, A., Munz, M., Molzow, I., Wilhelm, I., Wiesner, C. D., \& Baving, L. (2013). Sleep promotes consolidation of emotional 
memory in healthy children but not in children with attention-deficit/ hyperactivity disorder. PLOS ONE, 8(5), e65098.

Rajaram, S. (1993). Remembering and knowing: Two means of access to the personal past. Memory \& Cognition, 21(1), 89-102.

Randler, C. (2013). German version of the reduced MorningnessEveningness Questionnaire (rMEQ). Biological Rhythm Research, 44(5), 730-736.

Ranganath, C. (2010). Binding items and contexts: The cognitive neuroscience of episodic memory. Current Directions in Psychological Science, 19(3), 131-137.

Rauchs, G., Feyers, D., Landeau, B., Bastin, C., Luxen, A., Maquet, P., \& Collette, F. (2011). Sleep contributes to the strengthening of some memories over others, depending on hippocampal activity at learning. The Journal of Neuroscience, 31(7), 2563-2568.

Richter-Levin, G., \& Akirav, I. (2003). Emotional tagging of memory formation-in the search for neural mechanisms. Brain Research Reviews, 43(3), 247-256.

Rimmele, U., Davachi, L., Petrov, R., Dougal, S., \& Phelps, E. A. (2011). Emotion enhances the subjective feeling of remembering, despite lower accuracy for contextual details. Emotion, 11(3), 553-562.

Ritchey, M., Dolcos, F., \& Cabeza, R. (2008). Role of amygdala connectivity in the persistence of emotional memories over time: An eventrelated fMRI investigation. Cerebral Cortex, 18(11), 2494-2504.

Rosales-Lagarde, A., Armony, J. L., del Río-Portilla, Y., Trejo-Martínez, D., Conde, R., \& Corsi-Cabrera, M. (2012). Enhanced emotional reactivity after selective REM sleep deprivation in humans: An fMRI study. Frontiers in Behavioral Neuroscience, 6, 25.

Rugg, M. D., Otten, L. J., \& Henson, R. N. A. (2002). The neural basis of episodic memory: Evidence from functional neuroimaging. Philosophical Transactions of the Royal Society B, 357(1424), 1097-1110.

Saletin, J. M., Goldstein, A. N., \& Walker, M. P. (2011). The role of sleep in directed forgetting and remembering of human memories. Cerebral Cortex, 21(11), 2534-2541.

Schabus, M., Dang-Vu, T. T., Albouy, G., Balteau, E., Boly, M., Carrier, J., ... Maquet, P. (2007). Hemodynamic cerebral correlates of sleep spindles during human non-rapid eye movement sleep. Proceedings of the National Academy of Sciences of the United States of America, 104(32), 13164-13169.

Schönauer, M., Pawlizki, A., Köck, C., Gais, S. (2014). Exploring the Effect of Sleep and Reduced Interference on Different Forms of Declarative Memory. Sleep, 37(12), 1995-2007.

Schreiner, T., \& Rasch, B. (2015). Boosting vocabulary learning by verbal cueing during sleep. Cerebral Cortex, 25(11), 4169-4179.

Sharot, T., \& Phelps, E. (2004). How arousal modulates memory: Disentangling the effects of attention and retention. Cognitive, Affective, \& Behavioral Neuroscience, 4(3), 294-306.

Sharot, T., \& Yonelinas, A. P. (2008). Differential time-dependent effects of emotion on recollective experience and memory for contextual information. Cognition, 106(1), 538-547.

Snodgrass, J. G., \& Corwin, J. (1988). Pragmatics of measuring recognition memory: Applications to dementia and amnesia. Journal of Experimental Psychology: General, 117(1), 34-50.

Staresina, B. P., Bergmann, T. O., Bonnefond, M., van der Meij, R., Jensen, O., Deuker, L., ... Fell, J. (2015). Hierarchical nesting of slow oscillations, spindles and ripples in the human hippocampus during sleep. Nature Neuroscience, 18(11), 1679-1686.

Staresina, B. P., \& Davachi, L. (2009). Mind the gap: Binding experiences across space and time in the human hippocampus. Neuron, 63(2), 267-276.

Sterpenich, V., Albouy, G., Boly, M., Vandewalle, G., Darsaud, A., Balteau, E., ... Maquet, P. (2007). Sleep-related hippocampo-cortical interplay during emotional memory recollection. PLOS Biology, $5(11)$, e 282

Sterpenich, V., Albouy, G., Darsaud, A., Schmidt, C., Vandewalle, G., Vu, T. T. D., ... Balteau, E. (2009). Sleep promotes the neural reorganization of remote emotional memory. Journal of Neuroscience, 29(16), 5143-5152.

Stickgold, R., \& Walker, M. P. (2013). Sleep-dependent memory triage: Evolving generalization through selective processing. Nature Neuroscience, 16(2), 139-145.

Studte, S., Bridger, E., \& Mecklinger, A. (2015). Nap sleep preserves associative but not item memory performance. Neurobiology of Learning and Memory, 120, 84-93.

Studte, S., Bridger, E., \& Mecklinger, A. (2017). Sleep spindles during a nap correlate with post sleep memory performance for highly rewarded word-pairs. Brain and Language, 167, 28-35.

Tononi, G., \& Cirelli, C. (2003). Sleep and synaptic homeostasis: A hypothesis. Brain Research Bulletin, 62(2), 143-150.

Tucker, M. A., Tang, S. X., Uzoh, A., Morgan, A., \& Stickgold, R. (2011). To sleep, to strive, or both: How best to optimize memory. PLOS ONE, 6(7), e21737.

Tukey, J. W. (1977). Exploratory data analysis. Boston, MA: Addison-Wesley.

Unsworth, N., Fukuda, K., Awh, E., \& Vogel, E. K. (2014). Working memory and fluid intelligence: Capacity, attention control, and secondary memory retrieval. Cognitive Psychology, 71, 1-26.

van Ast, V. A., Cornelisse, S., Meeter, M., Joëls, M., \& Kindt, M. (2013). Time-dependent effects of cortisol on the contextualization of emotional memories. Biological Psychiatry, 74(11), 809-816.

van der Helm, E., Gujar, N., Nishida, M., \& Walker, M. P. (2011). Sleepdependent facilitation of episodic memory details. PLOS ONE, 6(11), e27421.

van Dongen, E. V., Thielen, J. W., Takashima, A., Barth, M., \& Fernández, G. (2012). Sleep supports selective retention of associative memories based on relevance for future utilization. PLOS ONE, 7(8), e43426.

Ventura-Bort, C., Löw, A., Wendt, J., Moltó, J., Poy, R., Dolcos, F., ... Weymar, M. (2016). Binding neutral information to emotional contexts: Brain dynamics of long-term recognition memory. Cognitive, Affective, \& Behavioral Neuroscience, 16(2), 234-247.

Vyazovskiy, V. V., Borbély, A. A., \& Tobler, I. (2002). Interhemispheric sleep EEG asymmetry in the rat is enhanced by sleep deprivation. Journal of Neurophysiology, 88(5), 2280-2286.

Wagner, U., Gais, S., \& Born, J. (2001). Emotional memory formation is enhanced across sleep intervals with high amounts of rapid eye movement sleep. Learning \& Memory, 8(2), 112-119.

Walker, M. P., \& van der Helm, E. (2009). Overnight therapy? The role of sleep in emotional brain processing. Psychological Bulletin, 135(5), $731-748$.

Wang, B. (2014). Effect of time delay on recognition memory for pictures: The modulatory role of emotion. PLOS ONE, 9(6), e100238.

Watson, D., Clark, L. A., \& Tellegen, A. (1988). Development and validation of brief measures of positive and negative affect: The PANAS scales. Journal of Personality and Social Psychology, 54(6), 1063-1070.

Wechsler, D. (2008). Wechsler adult intelligence scale (4th ed.). San Antonio, TX: Pearson.

Werner, G. G., Schabus, M., Blechert, J., Kolodyazhniy, V., \& Wilhelm, F. H. (2015). Pre-to postsleep change in psychophysiological reactivity to emotional films: Late-night REM sleep is associated with attenuated emotional processing. Psychophysiology, 52(6), 813-825.

Weymar, M., Löw, A., \& Hamm, A. O. (2011). Emotional memories are resilient to time: Evidence from the parietal ERP old/new effect. Human Brain Mapping, 32(4), 632-640.

Wiesner, C. D., Pulst, J., Krause, F., Elsner, M., Baving, L., Pedersen, A., ... Göder, R. (2015). The effect of selective REM-sleep deprivation on the consolidation and affective evaluation of emotional memories. Neurobiology of Learning and Memory, 122, 131-141.

Wilhelm, I., Diekelmann, S., Molzow, I., Ayoub, A., Mölle, M., \& Born, J. (2011). Sleep selectively enhances memory expected to be of future relevance. Journal of Neuroscience, 31(5), 1563-1569. 
Yonelinas, A. P. (2002). The nature of recollection and familiarity: A review of 30 years of research. Journal of Memory and Language, 46(3), 441-517.

Yonelinas, A. P. (2013). The hippocampus supports high-resolution binding in the service of perception, working memory and long-term memory. Behavioural Brain Research, 254, 34-44.
Yonelinas, A. P., \& Jacoby, L. L. (1994). Dissociations of processes in recognition memory: Effects of interference and of response speed. Canadian Journal of Experimental Psychology, 48(4), 516-535.

Yonelinas, A. P., \& Ritchey, M. (2015). The slow forgetting of emotional episodic memories: An emotional binding account. Trends in Cognitive Sciences, 19(5), 259-267. 